\title{
Automatic Seed Detection in On-Line Portal Images for Prostate Treatment
}

\author{
By \\ Véronique Audet

\begin{abstract}
A thesis submitted to
the Faculty of Graduate Studies and Research

in partial fulfillment of

the requirements for the degree of

Master of Computer Science

Ottawa-Carleton Institute for Computer Science

School of Computer Science

Carleton University

Ottawa, Ontario
\end{abstract}

December 16, 2002

(C) Copyright

2002, Véronique Audet 
National Library

of Canada

Acquisitions and

Bibliographic Services

395 Wellington Street Ottawa ON K1A ON4 Canada
Bibliothèque nationale du Canada

Acquisitions et services bibliographiques

395, rue Wellington

Ottawa ON K1A ON4

Canada
The author has granted a nonexclusive licence allowing the National Library of Canada to reproduce, loan, distribute or sell copies of this thesis in microform, paper or electronic formats.

The author retains ownership of the copyright in this thesis. Neither the thesis nor substantial extracts from it may be printed or otherwise reproduced without the author's permission.
L'auteur a accordé une licence non exclusive permettant à la Bibliothèque nationale du Canada de reproduire, prêter, distribuer ou vendre des copies de cette thèse sous la forme de microfiche/film, de reproduction sur papier ou sur format électronique.

L'auteur conserve la propriété du droit d'auteur qui protège cette thèse. $\mathrm{Ni}$ la thèse ni des extraits substantiels de celle-ci ne doivent être imprimés ou autrement reproduits sans son autorisation.

\section{Canadä̀}


The undersigned recommend to

The Faculty of Graduate Studies and Research acceptance of the thesis

\title{
Automatic Seed Detection in On-Line Portal Images for Prostate Treatment
}

\author{
submitted by \\ Véronique Audet \\ in partial fulfillment of the requirements for \\ the degree of Master of Computer Science
}

\section{Frar olhe}

Dr. Frank Dehne

(Director, School of Computer Science)
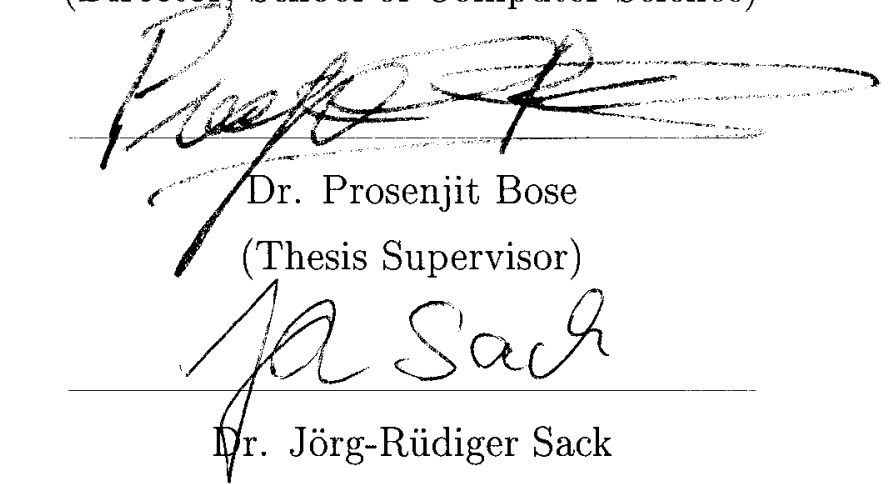

(Thesis Supervisor)

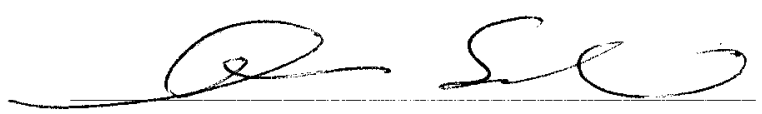

Dr. Janos Szanto

(Thesis Supervisor)

Carleton University

December 2002 


\section{Abstract}

External radiotherapy is one of the most common treatments for prostate cancer. Conformal Radiation Therapy (CRT) is a specific subset of radiation therapy aimed at preserving normal tissue, while controlling the tumour locally. There are uncertainties associated with radiotherapy: setup variations, patient motion and organ motion. In order for CRT to be affective, the prostate position must be known at treatment time, for proper dose delivery. Electronic Portal Imaging Devices (EPIDs) make use of the radiation beam to obtain portal images. These images help determine tumour position. To visualize the prostate on plain X-ray, three gold seeds are implanted into the prostate. In this thesis, a simple and fast automatic seed detection algorithm is presented, permitting the localization and tracking of the prostate during treatment. The tests indicate that in all cases considered the seeds are properly detected in portal images without any human intervention. 


\section{Acknowledgments}

I would like to start by expressing my sincere gratitude to my supervisors, Dr. Bose, Dr. Sack and Dr. Szanto for their time, generosity and collaborations. It has been interesting, motivating and fun to work with you. Your assistance is greatly appreciated. Thank you.

I also want to thank Dr. Nussbaum and M. Whitehead for their interest and beneficial discussions.

Je tiens à remercier ma famille pour leur soutien moral tout au long de mes études. Leurs encouragements et inspirations sont bien appréciés. Merci.

A thank you to all my friends who have been supportive and encouraging, particularly Marcus, Elizabeth, Josée, Margaret, Ian and Toby. Thank you for your ears on hard times and your laughs on great times.

Finally, I want to thank Jean Pouliot and Michèle Aubin from the department of Radiation Oncology at the University of California San Francisco for supplying us with images to test the algorithm presented in this thesis. 


\section{Contents}

1 Introduction $\quad 1$

1.1 Motivation . . . . . . . . . . . . . . . . 1

1.2 Thesis Contribution . . . . . . . . . . . . . . . 2

1.3 Thesis Outline ...................... 3

2 Prostate Cancer 4

2.1 Overview of Prostate Cancer . . . . . . . . . . . . . . . 4

2.2 Prostate Cancer Treatment . . . . . . . . . . . . . 6

2.2.1 Treatment Phases . . . . . . . . . . . . . 6

2.2.2 Radiopaque Markers ............... 6

2.2.3 Conformal Radiation Therapy . . . . . . . . . . . 7

2.2.4 Target Volumes . . . . . . . . . . . . . 8

2.2.5 Treatment Planning ............... 8

2.2 .6 Treatment Delivery . . . . . . . . . . . . . 9

2.3 Positioning Uncertainties . . . . . . . . . . . . . . . . . 13

2.3.1 Immobilization Devices . . . . . . . . . . . . . . . . 13

2.3.2 Prostate Motion . . . . . . . . . . . . . . . . . . 14

3 Imaging System $\quad 16$

3.1 Visualization of the Prostate . . . . . . . . . . . . 16

3.2 Portal Films . . . . . . . . . . . . . . . . . 17

3.2.1 Problems .......................... 17

3.3 Electronic Portal Imaging Devices . . . . . . . . . . . . . . . 17 
3.3.1 Mirror-Based Video System . . . . . . . . . . . . 18

3.3.1.1 Image Capturing . . . . . . . . . . . . . . 18

3.3.1.2 Properties ................... 19

3.3.1.3 Sources of Image Degradation . . . . . . . . . . . 19

3.3.1.4 Film vs Portal Images . . . . . . . . . . . . . . 21

3.4 The Oblique Fields . . . . . . . . . . . . . . . . . 21

4 Literature Review $\quad 22$

4.1 Noise Reduction . . . . . . . . . . . . . . . . . . 23

4.2 Image Enhancement . . . . . . . . . . . . . . . 24

4.3 Automatic Correction of Geometric

Uncertainties .......................... 25

4.3.1 Automatic Setup Verification ............. 25

4.3.2 Automatic Prostate Detection . . . . . . . . . . . . . 27

5 Seed Detection Algorithm $\quad 30$

5.1 Fundamental Problems . . . . . . . . . . . . . . . . . 31

5.2 Preliminaries . . . . . . . . . . . . . . . . . 32

5.2 .1 The Region Of Interest . . . . . . . . . . . . . . 32

5.2 .2 The Seed Geometry . . . . . . . . . . . . . . 33

5.3 Algorithm Steps . . . . . . . . . . . . . . . . . . 34

5.3 .1 Seed Intensity Ranges . . . . . . . . . . . . . 35

5.3 .2 Geometry Verification . . . . . . . . . . . . 39

5.3 .3 Center Of Gravity . . . . . . . . . . . . . 43

5.3 .4 Intra Seed Distances . . . . . . . . . . . . . 43

5.4 Seed Detection Failure . . . . . . . . . . . . . . . 44

6 Implementation and Experiments $\quad 46$

6.1 Test Cases . . . . . . . . . . . . . . . . . . . . . . . 46

6.2 Results . . . . . . . . . . . . . . . . . . . 47

6.3 Additional Testing . . . . . . . . . . . . . . . . . 52

6.4 Resources and Measures ................. 54 
6.4 .1 Space requirements . . . . . . . . . . . . . 54

6.4 .2 Running time ................... 55

6.4 .3 Clinical Time .................. 56

7 Conclusion and Future Work $\quad 57$

7.1 Conclusion . . . . . . . . . . . . . . . 57

7.2 Future Work . . . . . . . . . . . . . . . 57 


\section{List of Figures}

2.1 The prostate and neighbouring organs. . . . . . . . . . . 4

2.2 3D Conformal Radiation Therapy of the prostate. . . . . . . . . . 7

2.3 Simulation film of the prostate taken during treatment planning: \#1Base of prostate, \#2-Posterior wall of prostate and \#3-Apex of prostate. 9

2.4 Multi-Leaf Collimator (MLC) . . . . . . . . . . . . 10

2.5 Six fields of radiation of the prostate (target) . . . . . . . . . . 11

2.6 Beam's Eye View (BEV) of a field of the prostate. . . . . . . . . . 12

3.1 Port Film and Electronic Portal Image. . . . . . . . . . . . . . 16

3.2 Mirror-Based Video System. . . . . . . . . . . . . . . . . 18

3.3 Light Emission. . . . . . . . . . . . . . . . . . 20

5.1 Left Anterior Oblique (LAO) original image and contrast enhanced image. . . . . . . . . . . . . . . . . 31

5.2 Region of Interest (ROI) over seed $3 . \ldots . \ldots 33$

5.3 Reconstructed seed images: 1) original 2) smoothed 3) contrast enhanced and 4) ideal detection. . . . . . . . . . . . . . 35

5.4 Flow Chart of algorithm steps to detect one seed. . . . . . . . . . . 36

5.5 Intensity ranges for two seeds. . . . . . . . . . . . . . . 38

5.6 Seed shape, size and orientation. . . . . . . . . . . . . . 38

5.7 Seed Projection from the isocenter plane. . . . . . . . . . . . . . . . 39

5.8 Detected Polygon with a one pixel hole. . . . . . . . . . . . . . . . 40

5.9 Possible circle projections. . . . . . . . . . . . . . . . . . 41

5.10 Rejected seed geometry. . . . . . . . . . . . . . . . . 41 
5.11 Seed geometry: acceptable in A) and C), not acceptable in B) and D). 42

5.12 Original image, image with detected seeds and image with CoGs. . . 43

$5.13 \mathrm{LAO}$ and RAO with intra-seed distances. . . . . . . . . . . . . 44

6.1 Image with ROIs placed close to the field edge and the corresponding contrast enhanced image. . . . . . . . . . . . . . . 47

6.2 Detected seed values on iteration $1 \ldots \ldots \ldots \ldots \ldots$

6.3 Detected seed values on iteration $2 \ldots \ldots \ldots \ldots \ldots$

$6.43 \mathrm{x} 3$ blocks overlapping different seeds. . . . . . . . . . . 50

6.5 Edge pixel intensity graph of the corresponding horizontal line in the

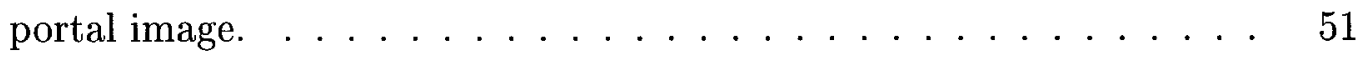

6.6 ROI values placed close to a field edge. . . . . . . . . . . . . 51

6.7 Original seeds with corresponding smoothed seed. . . . . . . . . 53

6.8 Lateral field image with corresponding contrast enhanced image. . . . 53

6.9 Detection of seed 3 placed close to the field edge and the hip bone in a lateral field image. . . . . . . . . . . . . . . 54

7.1 Field Edge Detection. . . . . . . . . . . . . . . . . . . 59 


\section{List of Tables}

5.1 Steps of Algorithm. . . . . . . . . . . . . . . . 36

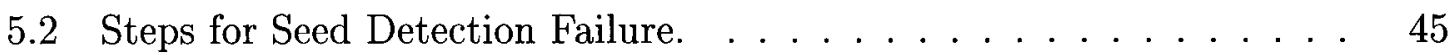

6.1 Results with test case (i) - ROI overlapping a seed. . . . . . . . 49

6.2 Results with test case (ii) - ROI overlapping a background region. . . 50

6.3 Seed detection failures in smoothed images. . . . . . . . . . 52

6.4 Running time of the seed detection algorithm in 6 portal images. . . . 56 


\section{List of Acronyms}

$\begin{array}{ll}\text { AD } & \text { Analog-to-Digital } \\ \text { AHE } & \text { Adaptive Histogram Equalization } \\ \text { BCM } & \text { Beam Center Marker } \\ \text { BEV } & \text { Beam's Eye View } \\ \text { CLAHE } & \text { Contrast Limited Adaptive Histogram Equalization } \\ \text { CT } & \text { Computer Tomography } \\ \text { CTV } & \text { Clinical Target Volume } \\ \text { CRT } & \text { Conformal Radiation Therapy } \\ \text { DICOM } & \text { Digital Imaging and Communication in Medicine } \\ \text { DRR } & \text { Digital Reconstruction Radiography } \\ \text { EPID } & \text { Electronic Portal Imaging Device } \\ \text { FPE } & \text { Field Placement Error } \\ \text { GTV } & \text { Gross Tumour Volume } \\ \text { HE } & \text { Histogram Equalization } \\ \text { LAO } & \text { Left Anterior Oblique Left } \\ \text { LoG } & \text { Laplacian of Gaussian } \\ \text { LPO } & \text { Left Posterior Oblique } \\ \text { MEK } & \text { Marker Extraction Kernel } \\ \text { MLC } & \text { Multi-Leaf Collimator } \\ \text { MRI } & \text { Magnetic Resonance Imaging } \\ \text { ORCC } & \text { Ottawa Regional Cancer Center } \\ \text { PTV } & \text { Planning Target Volume } \\ \text { RAO } & \text { Right Anterior Oblique } \\ \text { ROI } & \text { Region Of Interest } \\ \text { RPO } & \text { Right Posterior Oblique } \\ \end{array}$


SAHE Selective Adaptive Histogram Equalization

SHE Selective Histogram equalization

SNR Signal-to-Noise Ratio

UCSF University of California San Francisco

UMCU University Medical Center Utrecht 


\section{Chapter 1}

\section{Introduction}

\subsection{Motivation}

Prostate cancer is one of the leading cancers in North American men. The treatment of choice for localized diseases such as prostate cancer, is external radiation therapy. During radiation treatment delivery, there are three major categories of geometric uncertainties: setup errors, patient motion and organ motion. During radiation treatment, the deviation of the prostate and of the patient from the prescribed position can be determined with images taken with Electronic Portal Imaging Devices (EPIDs). Electronic portal images are captured with high energy radiation resulting in images that are noisy, have low contrast and poor sharpness. Since, the prostate and surrounding organs are soft tissue, they are not visible in portal images captured with EPIDs. To visualize the prostate in these images, three fiducial gold seeds are placed in the prostate. If the seeds can be localized at treatment time, the location of the prostate can be determined, thereby reducing setup uncertainties.

Prostate motion is mainly caused by the motion of surrounding organs: the bladder and the rectum. To compensate for the motion of the prostate, a margin around the radiation target is explicitly defined creating larger field sizes for radiation. However, these margins can increase the risk of radiation injury to the surrounding healthy organs. The injuries can be severe and often devastating to the patient. Automatically localizing the prostate gland at treatment time can considerably reduce the added 
margin and hence, reduce the side effects of radiation treatment. At the Ottawa Regional Cancer Center (ORCC), the width of the margin is set to $1 \mathrm{~cm}$. If the tumour could be located accurately at treatment time, the width would be decreased to 0.5 $\mathrm{cm}$, lessening the irradiated volume by approximately $40 \%$. Furthermore, a dose escalation can be applied to the tumour achieving better treatment outcome. Unless stated otherwise, all specific techniques mentioned in this thesis are those used at the ORCC.

Due to possible geometric uncertainties, the prostate changes in position throughout the course of radiotherapy. Therefore, the main goal of this thesis is to develop an efficient and accurate method to automatically detect the gold seeds, which allows us to accurately locate the prostate at treatment time. In the past decade, researchers have attempted to solve the problem of automatic target location using radiopaque markers and EPIDs. The methods include full image processing techniques and a considerable number of preprocessing steps. With the new portal imaging devices, the quality of portal images has been significantly improved to the point that without any preprocessing, the gold seeds are differentiable from the background intensities. In this thesis, we introduce a simple and less demanding image processing technique to automatically detect the fiducial seeds in portal images.

Problem 1.1 Given a portal image I, automatically detect the fiducial gold seeds $s_{i}$ in the image (for $i=1,2,3$ ) such that detection time and error is minimized.

\subsection{Thesis Contribution}

The contribution of this thesis is the design and implementation of a new seed detection algorithm improving upon previous methods due to its simplicity and ease of implementation. Furthermore, the algorithm presented requires a smaller number of steps in finding the fiducial seeds accurately, reducing the running time of the algorithm. No complicated image processing techniques are used, only simple histogram determination and the geometric definitions of the seeds are used. The algorithm is fast, accurate and simple permitting the thesis to possibly be used in clinical practice. The thesis offers a new image guided target technique for radiation 
treatment of prostate carcinoma, in attempt to decrease the side effects of radiation treatment. Moreover, the algorithm presents the potential of furthering the image guided technique to other types of cancers such as liver, pancreas and lung cancer. The material from this thesis was presented, in part, at the Visualization Imaging and Image Processing (VIIP) Conference [2].

\subsection{Thesis Outline}

The next chapter provides a summary of prostate cancer and possible treatments. An explanation of the treatment process utilized at the ORCC from the time the patient is diagnosed with prostate cancer to treatment delivery is presented.

A description of the two image capturing methods used during radiation treatment is given in Chapter 3. The major drawbacks along with the differences between the two systems are given.

Chapter 4 gives a review of the literature explaining the previous methods that attempted to solve the problem of geometric uncertainties occurring during the treatment of prostate cancer. The major topics include noise reduction methods for portal images, portal image enhancement techniques, setup verification methods and, the automatic detection of fiducial markers using EPIDs.

Chapter 5 outlines the seed detection algorithm. The major steps consist of locating the seed within a small region of the portal image, verifying the geometry of the detected seed, calculating the center of gravity of the seed and, calculating the distance relation between the three seeds in corresponding image pairs.

The test cases and results are given in Chapter 6 .

Chapter 7 concludes the thesis and presents a discussion on future work directions to explore before making the algorithm clinically acceptable. 


\section{Chapter 2}

\section{Prostate Cancer}

\subsection{Overview of Prostate Cancer}

Prostate cancer is one of the most common cancers in North American men. Cancer is a disease caused by the uncontrolled growth and possible spread of abnormal cells and, a tumour is a group of abnormal cells. As shown in Figure 2.1, the prostate is a small gland of the reproductive system located between the rectum and the bladder, both radiosensitive organs. It wraps around the first part of the urethra at the base of the bladder [50].

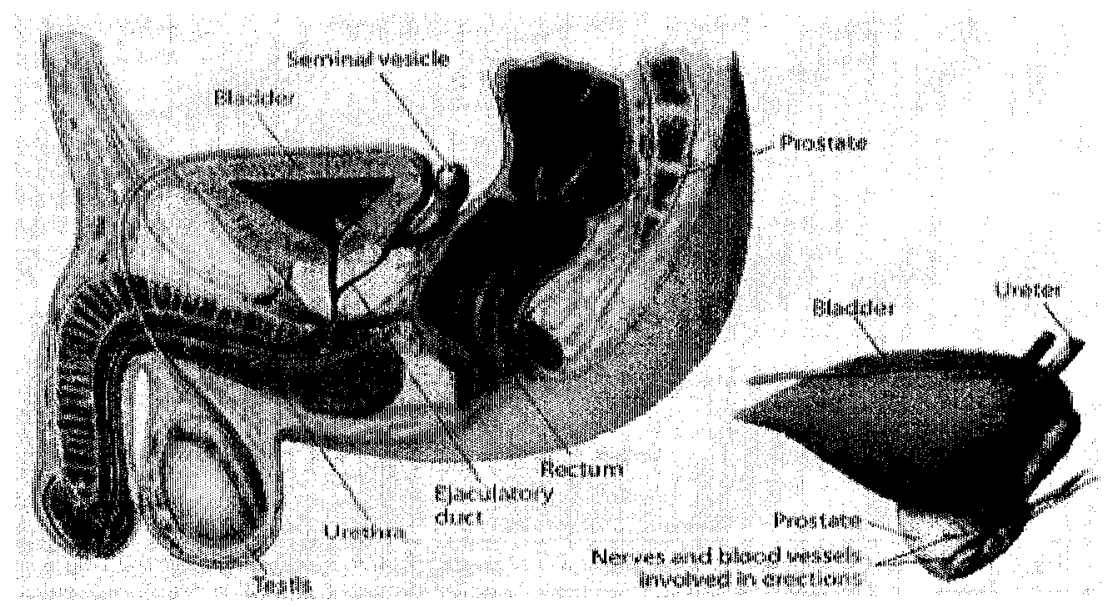

Figure 2.1: The prostate and neighbouring organs. 
Prostate cancer is a malignancy most commonly seen in older men. Patients undergo treatment when cancer cells grow inside the prostate and in more advance cases, when the cancer has spread to surrounding organs. The prostate moves and its position depends on the patient setup variations, on patient motion, on rectal and bladder filling and possibly other factors (e.g., patient breathing [24]).

Several treatment options are available for prostate cancer. The major treatments are surgery, radiation therapy, hormonal and/or chemotherapy [50]. The goal of surgery is to remove the prostate with all of the cancer. Radiation therapy tries to kill the tumour by exposing the cancer cells to high doses of radiation. There are two types of radiation treatment; external and internal. External radiation therapy radiates the prostate with a targeted beam of radiation from outside the body. Internal radiation therapy treats the tumour by implanting radioactive seeds inside the prostate, radiating the gland from inside the body, also known as brachytherapy. Hormonal therapy aims at stopping the reproduction of the hormone testosterone because testosterone can stimulate the growth of cancer cells. Finally, chemotherapy makes use of drugs to destroy active abnormal cells that are dividing rapidly. At the ORCC, the treatment of choice is external radiation therapy. The goal is to deliver enough radiation to eradicate the tumour while avoiding significant complications to the surrounding normal tissues. The most common side effect of radiation therapy in patients is critical rectal bleeding due to the radiation damage to the rectum.

The prostate is soft tissue and hence not visible on plain X-ray ${ }^{1}$. In order to visualize it on X-ray images, three small fiducial ${ }^{2}$ gold seeds are placed in the prostate prior to treatment. These seeds have a well defined geometric relationship relative to the contour points of the prostate.

\footnotetext{
${ }^{1} \mathrm{~A}$ photograph taken with $\mathrm{X}$-rays.

${ }^{2}$ Reference points in images taken prior and during treatment.
} 


\subsection{Prostate Cancer Treatment}

\subsubsection{Treatment Phases}

The following points give the ordered planification of prostate cancer treatment once the patient is diagnosed with prostate cancer. We briefly review these steps in the following sections.

1) Transrectal placement of fiducial markers.

2) CT scanning.

3) Making the immobilization devices.

4) Treatment Planning.

5) Treatment Delivery.

6) Setup Verification.

\subsubsection{Radiopaque Markers}

Radiopaque $^{3}$ markers are used in radiation treatment planning and in the verification process because of their high density, permitting them to be visualized on X-ray. The ORCC uses gold, a high density metal that resists the passage of X-ray efficiently. The seeds size is a cylinder of $0.6 \mathrm{~mm}$ in diameter by $3 \mathrm{~mm}$ in length and are kept in the prostate indefinitely. The seeds are placed transrectally under ultrasound guidance: in the apex, posterior wall and the prostate base by a qualified radiologist. These implanted radiopaque markers are used for target localization at treatment planning simulation and at treatment delivery.

These markers are used to visualize the prostate at all stages of radiotherapy. Furthermore, they permit the oncologists to verify accurate tumour targeting of the prostate during radiation treatment. This permits the use of more conformal radiation fields, reducing side effects like rectal bleeding by using narrower margins around the target. The position of the gold seeds identifies the current position of the prostate. Therefore, accurate location of the seeds is crucial.

\footnotetext{
${ }^{3}$ Not transparent to x-rays; appearing light/dark grey in images.
} 


\subsubsection{Conformal Radiation Therapy}

The lack of local control during treatment is the main reason for radiation failures. Conformal Radiation Therapy (CRT) is a specific subset of radiation therapy aimed at distributing the required radiation dose to closely match the shape of the target volume in order to avoid sensitive surrounding tissues [41]. Conforming to the shape of the prostate as closely as possible is the primary means of keeping the radiation from damaging other nearby healthy organs such as the bladder and the rectum. CRT decreases the probability of acute toxicity and long term morbidity. The development of 3-dimensional CRT allows for high accuracy in the shaping of the treatment volumes. The patient is treated with a course of fractionated treatments. The dose is delivered to a series of treatment fields that are individually shaped and delivered sequentially by a computer. Figure 2.2 demonstrates a patient lying on the treatment table while radiation is delivered to the target volume from six different angles.

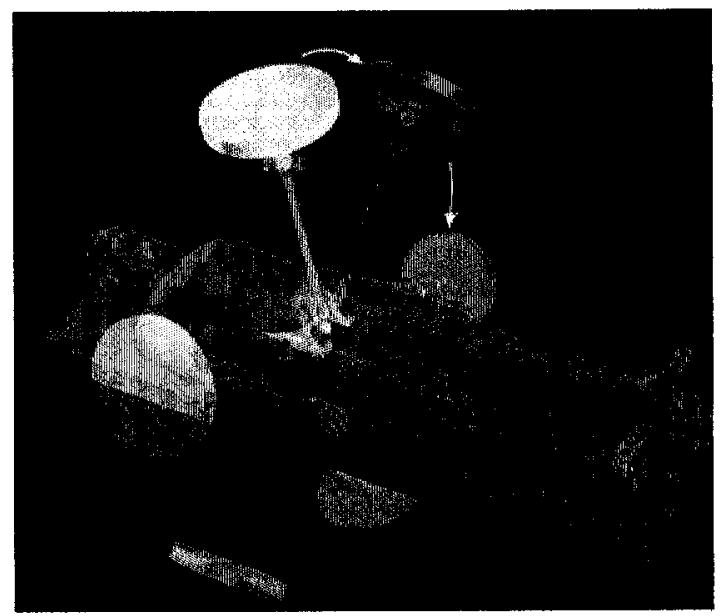

Figure 2.2: 3D Conformal Radiation Therapy of the prostate. 


\subsubsection{Target Volumes}

There are three main definitions used to evaluate the target of radiation volume $[30,41]$.

1) The Gross Target Volume (GTV) consists of the prostate gland.

2) The Clinical Target Volume (CTV) is based on the risk of microscopic spread of the disease. As a result, the CTV contains the GTV plus other possible tissues having the cancer.

3) The Planning Target Volume (PTV) considers the setup uncertainties such as prostate movement. The PTV then contains the CTV plus a margin around the CTV. The PTV is the target volume that is irradiated during radiation treatment.

\subsubsection{Treatment Planning}

The size, shape and position of the prostate inside the body are determined by taking Computer Tomography (CT) data of the prostate gland. The CT scan is performed by taking $5 \mathrm{~mm}$ thick slices through the prostate ${ }^{4}$. A large number of slices are taken to accurately delineate the GTV and possibly, the organs at risk surrounding the prostate such as the seminal vesicle. The radiation oncologist contours the prostate on all CT slices, which in turn is used to define the treatment fields.

Fiducial seeds are first used at the time of treatment planning simulation to define the treatment fields, which determine the shape of the target of radiation at a specific angle. Figure 2.3 gives an example of a simulation film where the three seeds are numbered. This process simulates the treatment by using a geometry similar to that of an accelerator ${ }^{5}$, but with much smaller energy beam to acquire diagnostic quality images. These images permit the oncologists to confirm the treatment and patient setup.

To accurately target the prostate with radiation, the beam is shaped to conform to the particular projection of the prostate with the help of a Multi-Leaf Collimator

\footnotetext{
${ }^{4}$ If the image guided technique presented in this thesis becomes mainstream, the CT thickness will be reduced to $2 \mathrm{~mm}$.

${ }^{5} \mathrm{~A}$ device that accelerates charged particles to high speed in order to produce nuclear reactions in a target.
} 


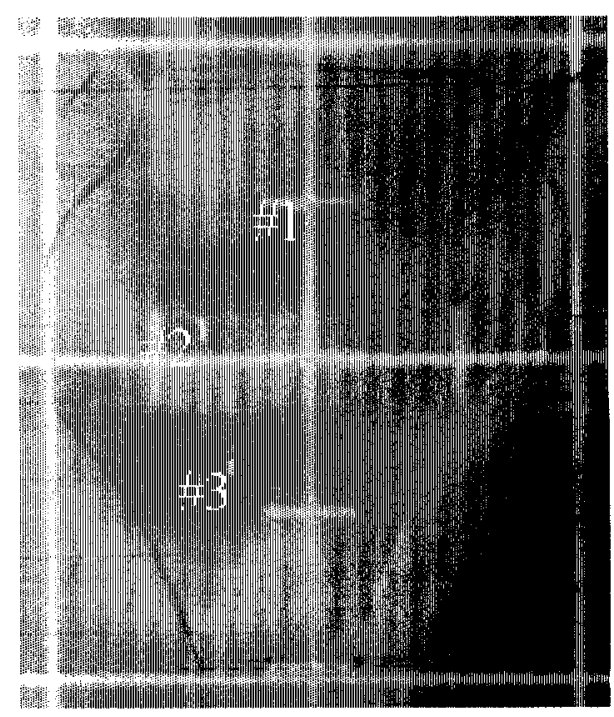

Figure 2.3: Simulation film of the prostate taken during treatment planning: \#1-Base of prostate, \#2-Posterior wall of prostate and \#3-Apex of prostate.

(MLC) system manufactured by Siemens; a standard accessory of a medical linear accelerator. As seen in Figure 2.4, this system consists of 29 pairs of $7 \mathrm{~cm}$ thick tungsten leaves, each having a width of $1 \mathrm{~cm}$. The left and right leaves move towards each other until the predesigned geometry of a particular field is achieved. Improvements in tumor and healthy tissue definition during simulation, translates into improved tumor control and in reduced toxicity levels during treatment delivery.

\subsubsection{Treatment Delivery}

As the patient is being irradiated, each fraction of radiation is delivered by different radiation beams. The head of the accelerator circles the patient and the patient is positioned on the treatment table such that the prostate is at the origin of rotation. The MLC shapes each beam to conform to the particular projection of the target volume. Since the bladder is positioned on top of the prostate (ventral direction) and the rectum on the bottom (dorsal direction), no direct radiation is given on the central vertical axis.

The isocenter is defined as the point in the center of each field, placed at the center of rotation of the head of the linear accelerator. Each leaf of the MLC is positioned 


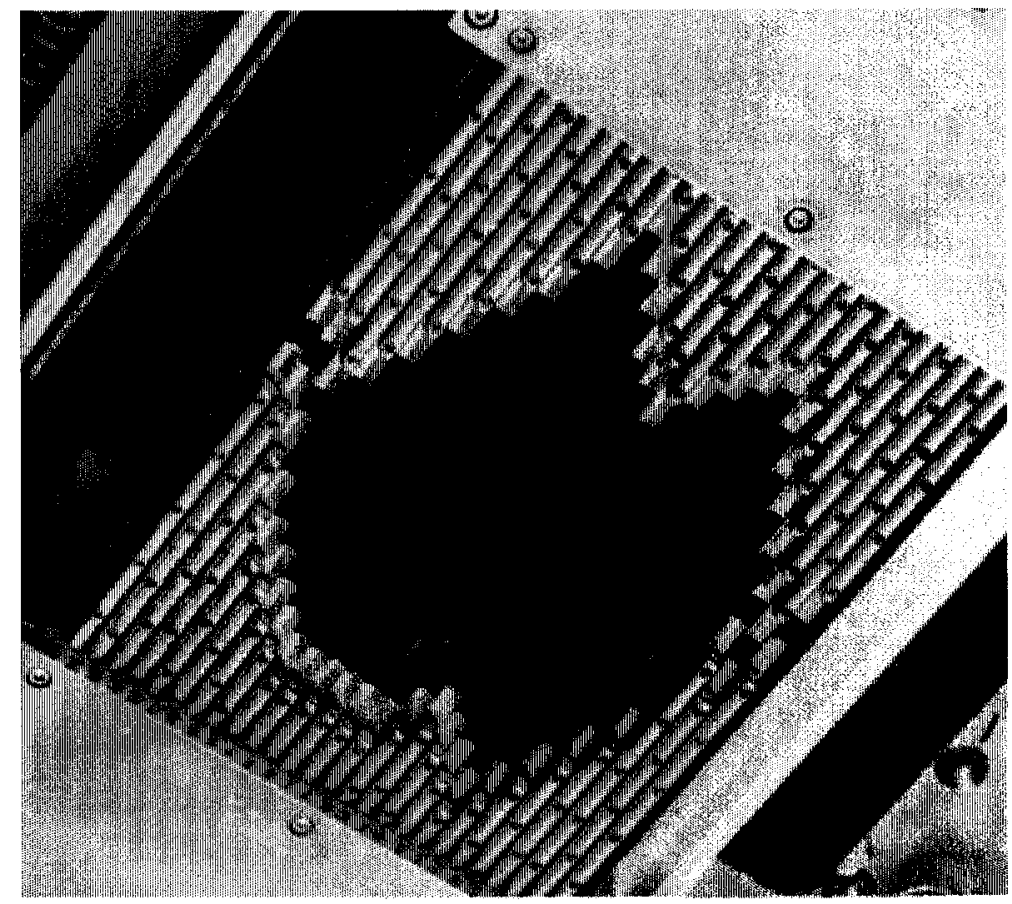

Figure 2.4: Multi-Leaf Collimator (MLC).

at a specific distance to the isocenter. Patients are treated using an isocentric 6-field conformal technique. Figure 2.5 is an image of a cross section of a patient lying on his back where the blue delineated shape is the prostate, the white regions represent the bony structures (hip bone) and the different fields are numbered from one to six in clockwise order.

For the small prostate fields most leaves are closed (first and last leaves) and the middle leaves are open following the Beam's Eye View (BEV) to capture the predefined geometry of the prostate field from a specific beam angle. The isocenter is usually at the center of the open middle leaf and is at the midpoint of the length (height) of the prostate field. Figure 2.6 gives an example of a $\mathrm{BEV}$ where $\mathrm{X}_{i}$ and $\mathrm{X}_{j}$ are the left and right leaves respectively, the associated distances are the distances to the isocenter; the red cross in the center of the field. In this example, the isocenter is placed in the center of leaf 15 . The Y1 and Y2 values are the distances to the isocenter from the top and bottom jaws. In Figure 2.6, the height of the prostate is $8.5 \mathrm{~cm}(\mathrm{Y} 1+\mathrm{Y} 2)$. Each of the leaf positions and length of the prostate are written 


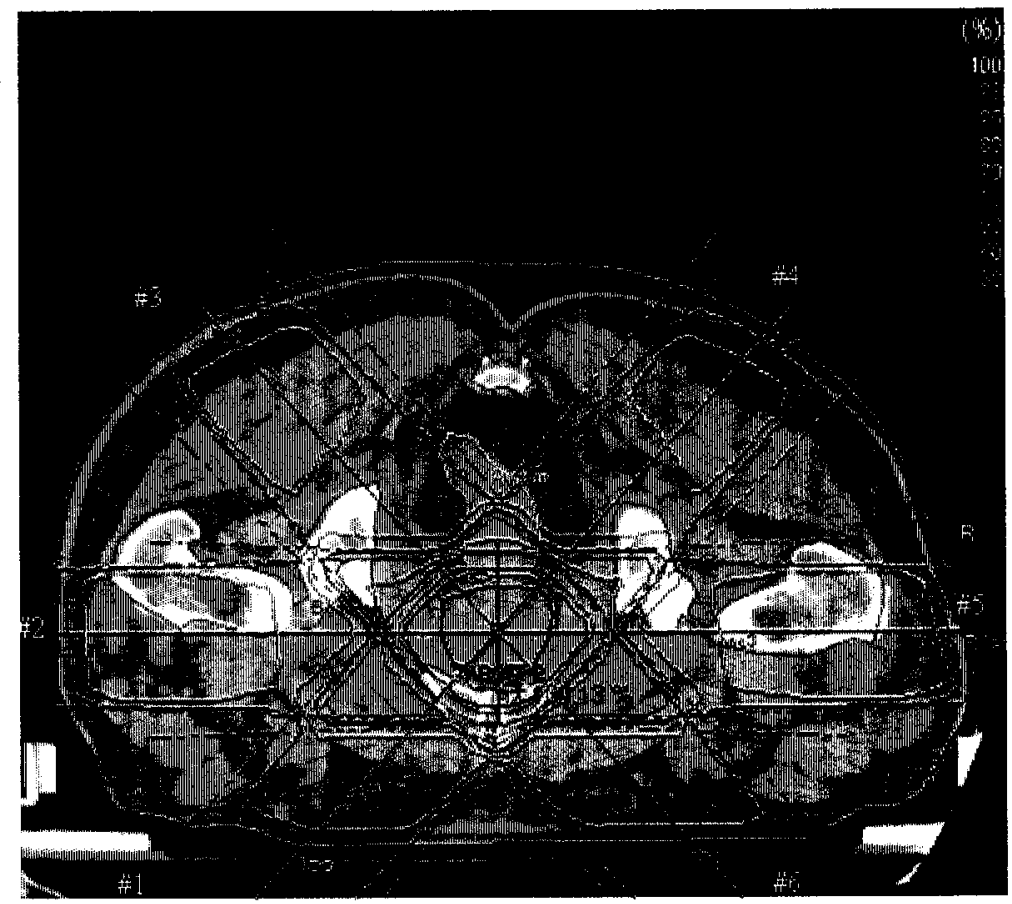

Figure 2.5: Six fields of radiation of the prostate (target).

to a file, keeping track of the treatment fields and setup information for each patient. 


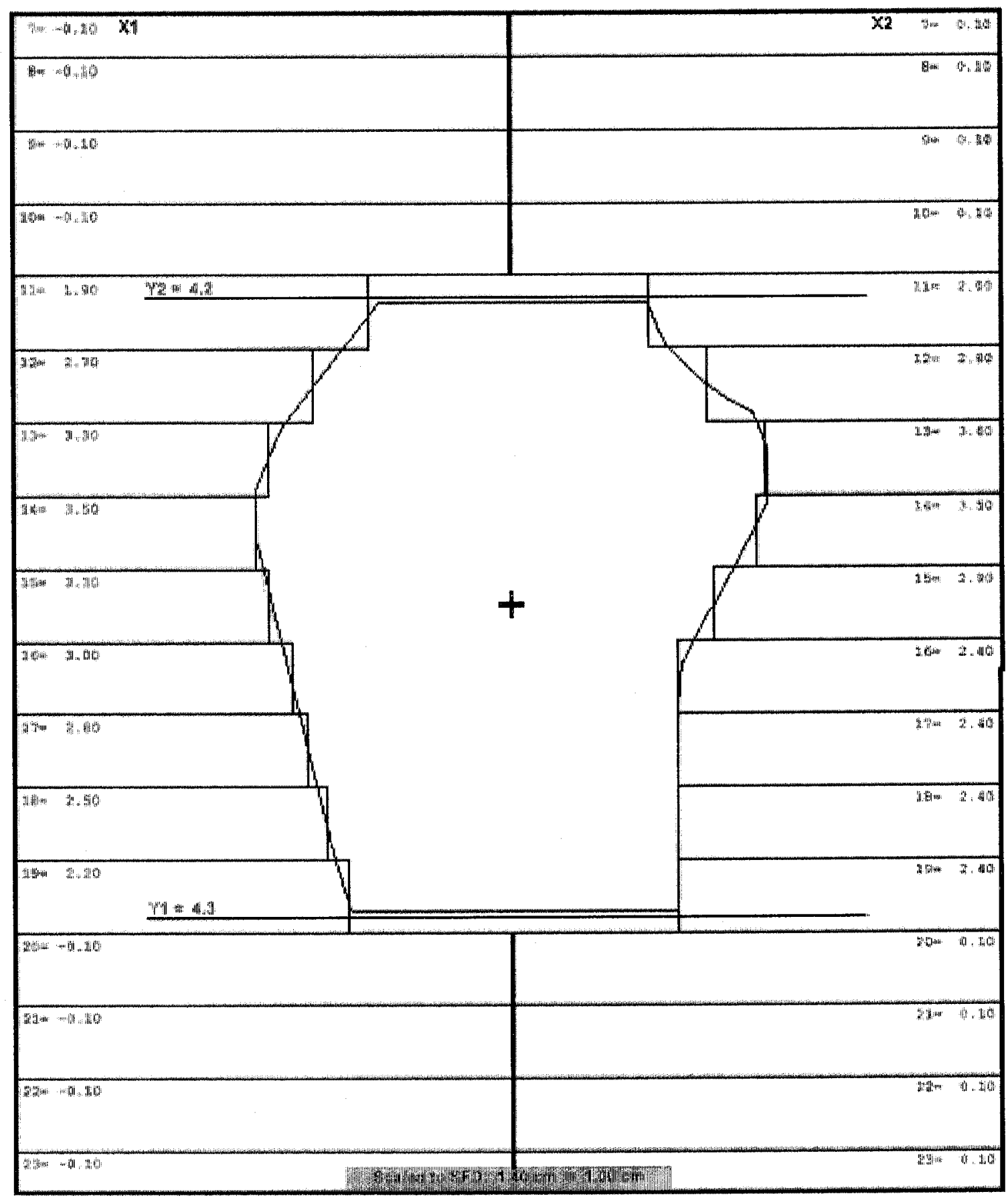

Figure 2.6: Beam's Eye View (BEV) of a field of the prostate. 


\subsection{Positioning Uncertainties}

Geometric precision in radiation treatment is crucial. Inaccurate positioning of the target can result in complications and recurrence of the cancer. To achieve conformal treatment of the tumour, exact patient positioning is essential. During treatment delivery, there are three types of geometric uncertainties: setup errors, patient motion and organ motion. The radiation target can shift in position on average from $1 \mathrm{~mm}$ up to $5 \mathrm{~mm}$ in any direction [23].

To ensure that the whole tumour gets treated, a margin is added to the prescribed target volume around the prostate creating larger field sizes for radiation. When determining these margins, geometric uncertainties become major considerations. If the margins are small, there is high risk of missing part of the tumour, however choosing too big a margin, increases the risk of radiation injury to the surrounding healthy organs.

These differences in target position relative to the treatment beam are called Field Placement Errors (FPE) [44]. The two major categories of FPEs are systematic and stochastic errors. A change in field position between treatments is considered a systematic error. The errors may be generated from a mistake in the conversion of the treatment planning field coordinates into the treatment delivery coordinates, incorrect radiation field alignment and/or the malfunctioning of radiation machinery. Stochastic errors differ between each radiation fraction and the main causes are patient motion and prostate motion.

\subsubsection{Immobilization Devices}

To reduce setup errors and patient movement during fractionated radiation treatment, patient immobilization devices are used. Patients are treated every day for six weeks. For conformal radiation delivery, the patient's position relative to the treatment beam needs to be invariant throughout the treatment process. Immobilization offers an accurate solution for reproducible patient setup over the course of treatment [40].

The use of immobilization significantly reduces the number of positioning errors greater than $5 \mathrm{~mm}$, potentially permitting the use of smaller treatment volumes [40]. 
There are three major patient immobilization systems for prostate treatment: the leg cushion, the alpha cradle and the Hipfix system [23]. The leg cushion technique immobilizes the knee to below the ankle in order to minimize the rotation of the legs and hip. The alpha cradle is a custom designed cushion, which extends from the mid-thorax to below the feet. Thirdly, the Hipfix is a thermoplastic shell molded to the patient's body around the pelvic area. The shell locks into a board on the sides and between the thighs of the patient on the treatment table. With the first two methods, the patients are placed supine (lie on their backs) and in the third method, the patients are placed prone (lie on their stomach). The Hipfix system largely reduces the setup deviations greater than $10 \mathrm{~mm}$ in all major axes in comparison to the other two methods [23].

To keep the patient invariant during treatment, the ORCC uses the Hipfix thermoplastic shells throughout simulation and treatment setup. With this system, the patients are placed prone except when the patients have back problems or are obese. The use of the Hipfix system reduces setup variability and helps in determining and minimizing the margins around the CTV resulting in decreased toxicity.

\subsubsection{Prostate Motion}

During treatment, prostate displacement must be taken into account. To optimize radiation therapy, many researchers have studied the range and primary directions of prostate movement and defined target margins accordingly $[3,11,23,24,25,26,43$, 46]. Study shows that the prostate moves in two predominant directions. There is little motion in the medial-lateral direction $(\mathrm{x})$, but greater in the cranial-caudal and in the anterior-posterior directions ( $\mathrm{y}$ and $\mathrm{z}$, respectively) $[3,11,13,46]$.

The physiological changes of rectal and bladder volume influence the position of the prostate and seminal vesicles [6]. Rectal filling has the strongest influence on prostate motion. Consequently, the relation between the contact of the rectum on the prostate with the amount prostate motion was investigated by many researchers. In particular, a research using cine Magnetic Resonance Imaging (MRI) was conducted to measure prostate motion with respect to the change in rectal diameter, with further considerations on the proximity of the rectum to the posterior border of the prostate 
gland [33].

There are two more probable causes in prostate motion. At the time of simulation when determining the prostate position and dimensions, urethrography ${ }^{6}$ and rectal barium are often used, and a significant change in prostate motion is detected $[25,26]$. The main reason for the displacement is the anxiety and discomfort associated to these procedures causing the patient to tense the pelvic muscles. A further cause is the respiratory induced movements influenced by the immobilization method used [24]. A significant respiratory associate prostate motion is present in patients immobilized prone with the Hipfix system. The prostate motion is most likely caused by the constraints induced by the shell resulting in an abdominal pressure transmitted to the pelvis.

Finding a systematic trend in the prostate gland displacement has no known solution, and therefore the displacement is taken to be random [46]. Immobilization devices help reduce deviations caused by setup errors and patient motion. However, to consider the variation due to organ motion, the inserted gold seeds must be detected in the images captured during radiation treatment. This thesis presents a solution in tracking the prostate at treatment time by automatically detecting the markers in electronic portal images.

\footnotetext{
${ }^{6} \mathrm{~A}$ dye injected intravenously during $\mathrm{X}$-ray studies of the urethra.
} 


\section{Chapter 3}

\section{Imaging System}

\subsection{Visualization of the Prostate}

Two types of images are taken during treatment to verify patient positioning relative to the treatment fields: portal films and electronic portal images. Portal films offer a hard copy of each treatment field before or after radiation treatment. Electronic portal images are digital 8-bit images and provide real-time information during treatment delivery. Figure 3.1 gives an example of the two types of images. They are inverted relative to each other with respect to the grey scale intensities.
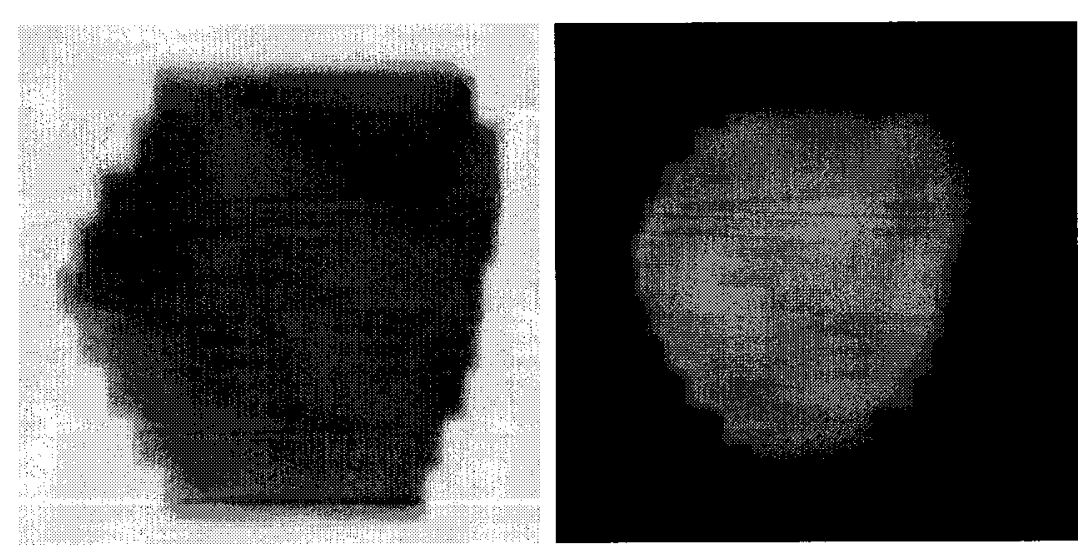

Figure 3.1: Port Film and Electronic Portal Image. 


\subsection{Portal Films}

Portal films are used to capture the placement of the treatment fields and are stored as hard copies. In radiotherapy, special silver-halide films (Kodak EC-L) are used and placed in a mobile cassette holder. There is a copper plate coated with phosphor in front of the film on the irradiated side. The radiation therapist places the cassette holder next to the patient close to the target so that the cassette holding the film is close to perpendicular to the central axis of the beam.

\subsubsection{Problems}

One important factor to consider is the position of the cassette that holds the film. If the radiation therapist places the cassette holder such that the plane of the cassette is not exactly perpendicular to the axis of the beam, the resulting images will be warped (projective 2-dimensional warping). As a result, the projection of the prostate field and consequently the field edges will not coincide with the MLC and radiation field information extracted from the patient's treatment file. Furthermore, the seed location and shape will not be accurately projected onto the film.

The cassette has no fix point in the room coordinate system because of the mobility of the cassette holder. Each time an image is captured, the cassette is placed next to the patient but not necessarily at the exact same position as for the previous images. In order to keep track of these changes in position, extrinsic camera parameters are needed.

\subsection{Electronic Portal Imaging Devices}

EPIDs are used during radiation treatment to improve the geometric accuracy of dose delivery. Portal imaging helps to detect errors and inaccuracies during treatment [44]. The images are produced using high-energy X-rays ${ }^{1}$, the exact beams that are used for treatment. The idea is to acquire quality images while minimizing patient exposure. The manufacturer of the medical linear accelerator (KD2 dual energy) is

\footnotetext{
${ }^{1} \mathrm{~A}$ stream of high-energy photons used for their penetrating power in radiotherapy.
} 
Siemens and the electronic portal images are captured with Siemens Beamview ${ }^{\text {plus }}$, a phosphor plate mirror-based video system.

\subsubsection{Mirror-Based Video System}

\subsubsection{Image Capturing}

The X-ray detector consists of a metal copper plate coated with a fluorescent phosphor screen. The X-ray interaction in the metal plate creates high-energy electrons that produce light in the phosphor screen. The metal copper plate has a dual purpose in blocking electrons scattered from the patient and producing a dose buildup in the phosphor screen to increase image brightness [49]. The light diffused through the screen is projected onto a mirror at a $45^{\circ}$ angle, which in turn directs it to a TV camera. Figure 3.2 is an illustration of the mirror-based video system. The video signal is digitized and viewed on the monitor of the workstation $[8,28]$. The detector is fully retractable, not interfering with patient setup.

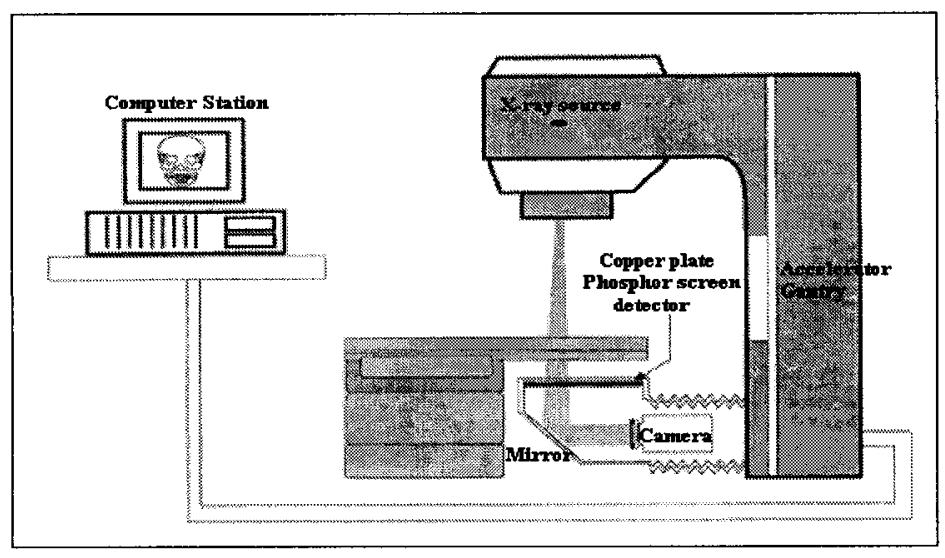

Figure 3.2: Mirror-Based Video System.

The source to isocenter distance is fixed at $100 \mathrm{~cm}$ and at $140 \mathrm{~cm}$ to the X-ray detector. The system acquires 8-bit images at 30 frames/second. The Beamview portal image size is $512 \times 480$ pixels and the spatial resolution of the imaging system is $0.55 \mathrm{~mm} /$ pixel in both the $\mathrm{x}$ and $\mathrm{y}$ directions. 


\subsubsection{Properties}

The list below provides the main properties found in images captured with EPIDs [14].

A) The field intensity values are higher (light) than the background (dark).

B) The background intensity values decrease as the distance to the field edge increases.

C) Noise is reasonably low in the background region.

D) Generally, local maxima of the gradient occurs at the field edges.

E) If anatomical structures appear at/near the field edge, the gradient values may maximize at the structures location and not at the field edge.

\subsubsection{Sources of Image Degradation}

In fractionated therapy, the dose is distributed between all fractions delivered compromising the quality of portal images. The radiation is delivered using high energy $\mathrm{X}$-rays and the exposure time is kept small to not increase the patient exposure by much. The use of high energy radiation beams with short image acquisition time results in inherently low contrast in portal images.

Another disadvantage is the amount of light collected by the camera. The light is scattered within the phosphor screen and emitted in all directions, as shown in Figure 3.3. Only a small fraction of light photons is captured by the camera's lens. Study show that only $0.01-0.1 \%$ of light reaches the TV camera [28].

The light output increases linearly with the phosphor thickness [49]. To improve the light collection, an increase in the thickness in the phosphor screen can therefore improve the overall image quality. Conversely, increasing the phosphor thickness intensifies the light photon scattering, resulting in a loss of the spatial resolution. Another possibility is to enlarge the camera lens in size in attempt to collect more of the light emitted. However, the larger size lens can generate problems; distortion which causes straight lines to appear curved at the edge of the field of view, a loss of spatial rendering due to spherical aberrations ${ }^{2}$ and, vignetting ${ }^{3}$ causing the image to

\footnotetext{
${ }^{2}$ Blurring of the image.

${ }^{3}$ Softening of edges along the periphery of the camera lens.
} 


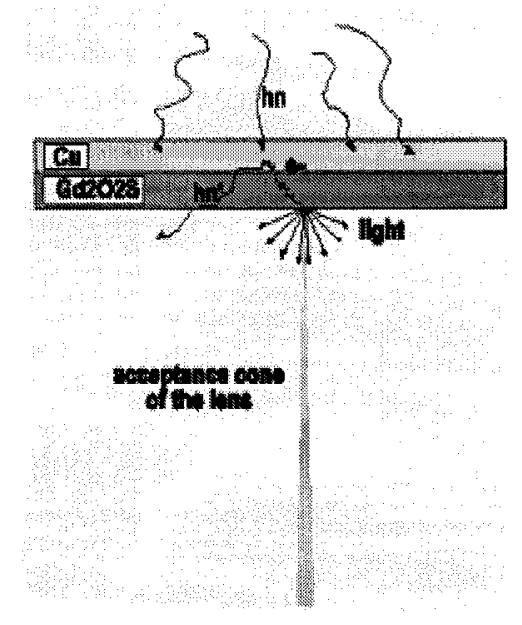

Figure 3.3: Light Emission.

look brighter at the center of the lens than at the edge [28, 29]. As a result, most of the improvements have been directed to improving the optics and reducing the noise produced by the electronics of the TV camera.

The noise generated by a video-based EPID is additive and in part depends on the acquisition time and energy of the beam [16]. The Signal-to-Noise Ratio (SNR) is low mainly caused by the large dark current ${ }^{4}$ generated by the electronics [8]. The two main causes in the increase of dark current are the degradation of the camera due to radiation damage and the increase in the temperature of the camera's components. These result in a gradual reduction of the video signal and eventually increasing the noise and loss of details in the images.

All considered, the major disadvantage in working with portal images is the inherently low contrast component making the problem of automatic seed detection more difficult. Recently, Siemens has developed a new version of Beamview ${ }^{\text {plus }}$ improving the quality of images thus, resulting in higher contrast between fiducial seeds and background intensities.

\footnotetext{
${ }^{4}$ Current that flows in the photo detector which degrades the image quality.
} 


\subsubsection{Film vs Portal Images}

The contrast and spatial resolution of films are better in comparison to electronic portal images. Films are placed in a cassette right next to the phosphor screen so that the distance between the detector and the image acquisition device is minimal, optimizing the conversion from X-ray to light. The films are designed to be most responsive to the light frequency generated in the phosphor. Consequently, the light output is maximized resulting in a smaller phosphor thickness, which in turn attains better spatial resolution.

Conversely, in the video-based portal imaging system the distance between the detector and the image acquisition device is quite large. The light must be reflected onto a mirror then captured by a camera causing flares and other artifacts. Due to the poor light collection, the phosphor thickness must be increased to capture more light causing photon scatter, which decreases the spatial resolution [49].

\subsection{The Oblique Fields}

During treatment, the gantry ${ }^{5}$ of the medical linear accelerator rotates about the target until it reaches the planned angle. Prostate cancer is treated using six conformal radiation fields. Portal images of these fields are taken when the beam's axis is at $45^{\circ}$ oriented to the patient's back and stomach, relative to the vertical axis (oblique projections) as well as when the axis is going through the patient's side in the horizontal plane (lateral projections).

This thesis analyzes orthogonal pairs of images, which are taken at perpendicular field projections. With the seed locations known in a pair of images, the location of these seeds in the room coordinate system can be calculated using linear algebra techniques, giving the location of the prostate in the patient's body at that time. The images may include Left Post Oblique (LPO) and Right Post Oblique (RPO) or Left Anterior Oblique (LAO) and Right Anterior Oblique (RAO) pairs. The thesis excludes the lateral views because of the prevalent bony structures (hip bone) and of the increase tissue thickness in that direction resulting in poorer image quality.

\footnotetext{
${ }^{5}$ Includes the collimator and the radiation source.
} 


\section{Chapter 4}

\section{Literature Review}

To consider the possible FPEs during treatment delivery, a set of margins are included in the PTV and to reduce FPEs, EPIDs are used. As mentioned in Chapter 2, there are three major geometric uncertainties during radiation treatment of prostate cancer. In attempt to correct set-up errors and patient motion, the patient's position is monitored with respect to the treatment beam by automatically detecting the bony structures and/or the field edges in the portal images. To correct deviations due to organ motion, the prostate must be automatically located by detecting the implanted gold seeds.

The difficulty in automatic feature detection in portal images captured with a video imaging system, is due to noise along with inherently low contrast between the image features and the background intensities. The poor quality of portal images has led to the study on the nature of noise to find a technique that will improve portal image quality. Image enhancement techniques attempt to emphasize features present in portal images. Therefore, before any feature detection algorithms are applied, noise reduction and contrast enhancement techniques are commonly used. In the past decade, researchers have attempted to solve the automatic seed detection problem using radiopaque markers and EPIDs. We review some of the relevant research below. 


\subsection{Noise Reduction}

Most noise removal techniques rely on some form of averaging, either locally or globally within the image. Statistically, noise can be considered as "outlying" data and averaging methods try to filter the outliers. The type of averaging that works best depends on the type of noise present.

There are two types of noise generated by a mirror-based video portal imaging system: random noise and fixed pattern noise [38, 39]. Random noise is categorized into three sub types. Quantum noise originates from the process of the conversion of electrons into photons, followed by the formation of a signal in the video camera, electronic noise is generated by the electronics of the system and thirdly, quantization noise. The camera captures several frames per second, in order to get all information in the frames, the final image is the result of the average of all frames used in the video. Quantization noise is introduced by integer arithmetic used in the frame averaging method and in the Analog-to-Digital (AD) converter [5, 38]. Frame averaging truncates real numbers to integers and the input to the $\mathrm{AD}$ converter quantizes the video signal by rounding off the input signal values. In portal images captured with a mirror-based video system, between the two quantization noise sources, the noise generated by the integer averaging has a greater effect in degrading image quality. Finally, fixed pattern noise is due to mechanical problems occurring in the phosphor screen, in the video camera and/or in the AD converter.

Many digital techniques exist to suppress noise in images. Temporal averaging and spatial filtering methods are commonly used [19]. Temporal averaging is a means of averaging all subsequent frames. The two major categories of spatial filtering methods are linear and non-linear. Linear methods have the effect of smoothing, hence degrading the spatial resolution in the image [19]. Non-linear techniques try to remedy this effect by reducing noise significantly without blurring important features. Consequently, non-linear systems are a more suitable approach in suppressing noise in medical images since the identification of anatomical structures and radiation field edges are sometimes crucial in patient setup verification. 


\subsection{Image Enhancement}

Once noise has been removed from an image, the next step consists of enhancing the areas of interest within the image. Preprocessing can help in automatic segmentation of anatomical features $[16,27]$. Accentuating the contrast between features in portal images improves the quality in the display. The most commonly used preprocessing steps are contrast enhancement techniques which modifies the image histogram values globally or locally. These methods facilitates a human observer such as the oncologist to review portal images and verify treatment setup. We give a review on enhancement methods below.

Different histogram modification techniques are used to amplify the contrast between features [18]. The disadvantage of using enhancement algorithms is the additional computation time along with the generation of noise. For relatively uniform images, global contrast techniques are optimal but local details are lost in non-uniform regions in the image. Local contrast histogram modifications are ideal for keeping details present in the image, but can be computationally expensive [18].

Histogram Equalization (HE) is a common method employed in image processing to increase the contrast between features in images. In the enhanced image, all intensity values are represented by approximately an equal number of pixels. The intensity values between features are spread out, making the intensity differences noticeable. Selective Histogram Equalization (SHE) is a different means of contrast enhancement that separately utilizes the HE method for the field region and for the surrounding background region [10].

The Adaptive Histogram Equalization (AHE) approach applies the above technique independently to subregions of the image [36]. Rather than working with the whole image intensity values, AHE calculates the local contrast of a region by using only surrounding pixel values. However, adaptive methods usually come with an interpolation process which is computationally expensive. Furthermore, they amplify noise in images because homogeneous regions are mapped to the local grey scale range. To evercome this, the amount of contrast can be limited with the Contrast Limited Adaptive Histogram Equalization (CLAHE) method; one of the most popu- 
lar contrast enhancement techniques in medical imaging $[35,36]$. This method clips the histogram so that the excess pixels are redistributed among all intensity values in the histogram. CLAHE can however reduce the detail level on the field edges [21]. A proposed solution is the Selective Adaptive Histogram Equalization (SAHE) method which is a modified version of the CLAHE algorithm by using overlapping subregions in the image. The method is selective because it applies the enhancement technique only to the pixels inside the field edge and adaptive because it employs the enhancement technique locally.

\subsection{Automatic Correction of Geometric Uncertainties}

Once the patient is ready for treatment, it is essential to verify the geometric alignment between the target and the radiation beam. To achieve proper dose delivery, accurate and reproducible placement of the treatment fields is crucial.

To measure the displacement of the patient's position relative to the predefined radiation beam position, the automatic detection of bony structures and field edges in portal images is used to compare the location of these features with the corresponding location in reference images. The difference in distances between the features give the deviations of setup errors and patient motion. To detect a change in position of the prostate, the implanted gold seed must be detected in the portal images.

\subsubsection{Automatic Setup Verification}

Theoretically, the landmarks of a patient in a simulation film (treatment planning) should coincide with the corresponding landmarks in the portal image (treatment delivery). If the landmarks do not match, a setup deviation is present and the patient's position must be corrected. Automatic setup verification is achievable with the combination of feature extraction and feature matching algorithms [13, 15, 20, 45, 47]. Feature extraction consists of detecting bony structures and/or field edges in portal images. Feature matching attempts to match and align the detected features in portal 
images to the corresponding features in reference images.

Numerous feature extraction algorithms exists. Approaches seen in the enhancement of anatomical details are the selective CLAHE method [20] and the Laplacian of a Gaussian (LoG) operator [15], a method similar to the second derivative which detects step edges in the images, the detection of creases in the images corresponding to the projected lines of structures with maximum bone thickness; a method that uses structure tensors [47] and, a top-hat transform [13, 47], a morphological transformation.

Automatic field edge detection methods include global thresholding and local gradient information. The edge can be approximated using global statistical information followed by an adjustment technique that estimates the edge with local gradient information. To delineate the contour of the treatment field, the knowledge on the direction of the local gradient maximum is used $[9,13,14,21]$. Some methods propose a field extraction algorithm based on morphological edge detection and segmentation techniques [48], and the use of a wavelet-based edge detector [34].

The feature matching process measures the deviation between the portal image and the reference image by calculating the displacement of corresponding landmarks. To compute the correlation between the portal image features and the reference image features, the method of choice is the chamfer matching algorithm [13, 34, 47]. This technique automatically aligns corresponding landmarks by finding the best fit between corresponding edge points by minimizing the distance between them. A reference image can be a simulation image or a Digital Reconstruction Radiography (DRR) image, both obtained during treatment planning. Some research studies use as the reference image the image taken during the first fraction of treatment $[12,15,34]$. However, if the field shape changes significantly between fractions, a better choice for reference images are simulation or DRR images. Furthermore, the quality of these images is significantly better than those taken during radiation treatment [34].

Another proposed method for position verification is a field edge matching technique [32]. The field edge in portal images is matched to the dosimetric field edge defined at treatment planning and the cross correlation between the detected polygons (field edges) is calculated. The best match between the field edges is determined 
by optimizing the cross correlation. The algorithm relies on leaf verification of the system hence not taking into account the errors in leaf positions at treatment time.

To test the accuracy of the system, the automatic results are compared to the results obtained with a trained human observer such as an experience radiographer $[13,47]$.

\subsubsection{Automatic Prostate Detection}

The underlying goal is to determine the location of the prostate as accurately as possible. This is done through the detection and location of radiopaque markers within portal images of the prostate. To date several groups of researchers have studied the problem of detecting markers in portal images. We briefly review each of their techniques.

One of the first studies used a sphere as a seed shape [4]. To test the ability of the system, four markers are placed in an anthropormorphic ${ }^{1}$ phantom. The markers are positioned as expected in a patient's prostate and the phantom is placed on the treatment table. The location of the markers are detected using a template-matching algorithm. They estimate the coordinates of the markers with a pair of orthogonal images. The procedures are tested with known phantom displacements and the system can detect deviations down to $1 \mathrm{~mm}$ translations and $1^{\circ}$ rotations.

Another approach is to use a specialized urethra catheter and a Beam-CenterMarker (BCM) [7]. The catheter contains radiopaque markers at specific positions and, the BCM consists of a tungsten indicator positioned in front of the beam projecting the isocenter in the portal images. Before treatment planning, the catheter is inserted into the patient, and at treatment planning, the isocenter is placed at one of the markers; the reference marker. The change in prostate position is detected by the difference in position between the reference marker and the isocenter. The system cannot detect the change in position of the prostate in the cranial-caudal (y) direction, but can calculate displacements down to $1 \mathrm{~mm}$ in the anterior-posterior (z) direction.

\footnotetext{
${ }^{1}$ Attribution of human characteristics to inanimate objects.
} 
Two groups of researchers have studied this problem more closely related to the topic presented in this thesis. The first group is at the University Medical Center Utrecht (UMCU) and the second at the University of California San Francisco (UCSF). The first group started extensive research on the visibility and automatic detection of gold seeds of different sizes in portal images [30]. Information is extracted from treatment planning $\mathrm{CT}$ data such that there is an a priory knowledge of the gold seeds approximate position, size and orientation. A urethra catheter containing seeds is placed inside the patient, and the rest of the seeds are placed on the patient's skin. To increase the contrast, display equalization methods are applied. To detect the seeds position in portal images, the group developed a Marker Extraction Kernel (MEK), a method based on chamfer matching techniques. The MEK calculates for each pixel in the image, the gradient value using neighbouring pixel values. The MEK response gives zero response for background constant intensities and maximal response for pixels positioned at a center of marker like shape (a seed). To test the accuracy of the system, a phantom was used.

The research progressed using a mirror-based video system with smaller seeds sizes [31]. The seeds are placed at equal distances to each other on the patient's skin at the beam exit and at the beam entry. To test the accuracy of detection, the seeds are placed on two perspex ${ }^{2}$ templates. One measured the detected distance between markers and the other measured the accurate detection in the orientation of the seeds. The group proposes to apply a median non-linear filter to smooth out noise in the image prior to calculating the MEK response. The detection success rate was higher for templates placed at the beam entry and the localization accuracy was in the worst case $0.6 \mathrm{~mm}$ for all markers used in the study.

Most recently, the group reviewed the MEK with smaller seed diameters using an amorphous-silicon flat panel imager [32]. Templates contained markers used in their two previous studies, but this time the markers are not placed at equal distances to each other. Lateral images are used in the study, making it more difficult to detect the seeds because of the apparent bony structures. The automatic seed location algorithm gave an overall accuracy of $0.4 \mathrm{~mm}$ and the detection time takes less than

\footnotetext{
${ }^{2} \mathrm{~A}$ transparent thermoplastic acrylic resin.
} 
1 second, giving a clinically acceptable detection success rate.

The second group studied the automatic detection of three radiopaque markers [37]. The search area in the images is reduced by using previous information available on the location of the markers. The seeds are identified in the enhanced portal images by finding the local minima created by the attenuation caused by the markers. These minima are given a weight corresponding to the data found in the contrast images. The detection algorithm makes use of known geometry between the three seeds. The accuracy is evaluated with a Plexiglas ${ }^{3}$ phantom containing three radiopaque markers placed on the treatment table. The results are validated with a neural network classifier. The overall accuracy is $90 \%$ and the detection algorithm takes under one second to compute.

This thesis presents a new seed detection algorithm that improves upon previous work in terms of the number of steps it requires to detect each seed. First, the algorithm searches for each seed locally, no full image processing is done. Secondly, no preprocessing techniques are applied, improving on the performance of the algorithm. Thirdly, the algorithm was tested with real data, no phantom was used. The images used in this thesis are taken during the radiation treatment of patients having prostate cancer. The algorithm presented works without any knowledge on the orientation of the seeds, only the approximate location of the seeds in a portal image is used. Moreover, the displacement of the prostate is calculated in all three major axis ( $\mathrm{x}, \mathrm{y}$, and $\mathrm{z}$ ) in terms of translation and rotation.

\footnotetext{
${ }^{3} \mathrm{~A}$ trademark used for a light, transparent, weather-resistant thermoplastic.
} 


\section{Chapter 5}

\section{Seed Detection Algorithm}

In clinical practice, the seed detection method must be fast, automatic, efficient and with a high degree of accuracy. Automatically detecting the prostate should not increase the time spent per patient on the treatment table or demand more work for the radiation therapist. For the seed detection algorithm to be clinically acceptable, the two major clinical requirements are as follows:

1) The validation of the prostate's position must be done in pseudo-real time (within one second).

2) The error must not exceed $1 \mathrm{~mm}$.

With the new technology of electronic portal imaging systems, image quality and contrast has been significantly improved over the first generation devices. Before any enhancement, most of the time the gold seeds are visible and simple contrast enhancement algorithms make the seeds clearly visible as seen in Figure 5.1.

If the seeds can be extracted with straightforward histogram manipulation, then the seed values in the original image must be differentiable from its surrounding pixels. This means that the deviation between a seed pixel and background pixel is greater than the deviation between two background pixels. This simple observation is the basis of the algorithm presented in this thesis. 

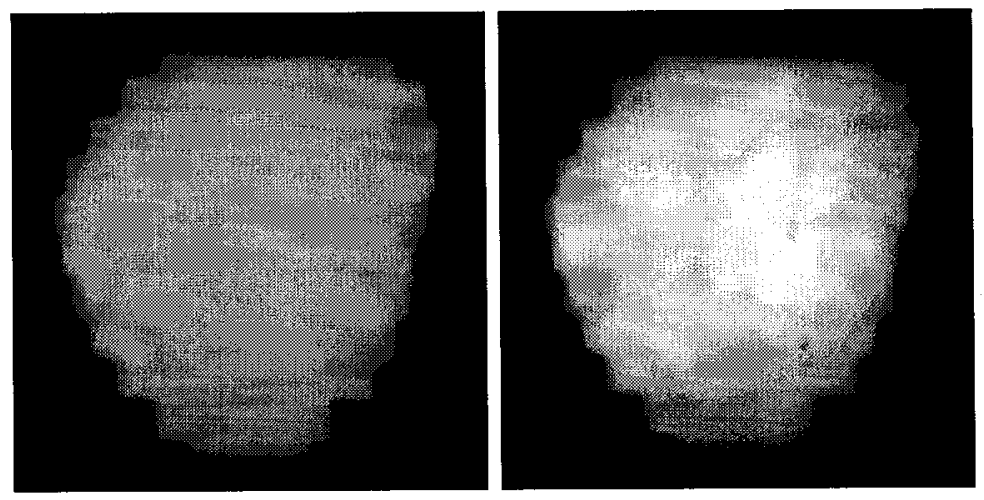

Figure 5.1: Left Anterior Oblique (LAO) original image and contrast enhanced image.

\subsection{Fundamental Problems}

The automatic seed detection algorithm must be reliable and consistent in finding the seeds accurately. It must be emphasized that a robust solution to the automatic seed detection is intrinsically difficult. There are three major reasons for that.

First, the contrast between features in X-ray images is limited by the nature of the interaction of photons within the patient. The dominant interaction at these energies is Compton scatter, which is independent of the atomic number, therefore the attenuation of the beam is not much different whether it passes through a gold seed or soft tissue. This results in inherently low contrast images. In diagnostic radiology, the energy of the beam is much lower, therefore the differential attenuation between the different atomic numbers is in fact amplified. For instance, using low energy X-rays the differential attenuation between soft tissue and bone is remarkable. At higher energies, the number of photons reaching the imaging plate is smaller so the image information is achieved with less photons during the image capturing process.

Secondly, the imaging system contains considerable system noise mainly caused by the following reasons. The small number of photons reaching the imaging plate, the low conversion efficiency of phosphor into visible light, the optical glare off the mirror, the optical scatter through the lens, the dark current from the CCD and, the noise generated by the electronics. The low contrast between features and the noise generated by the imaging system used, result in images having a low SNR. Due to the low contrast at high X-ray energies between a seed and the background, 
the difference between seed pixels and background pixels is small, making it difficult to find a threshold value that differentiates the seed from the background noise. In other words, the difference between these features and the difference between only background pixels is significantly close.

Thirdly, the size of a seed on portal images is generally just a few pixels. Increasing the seed size is not necessarily an optimum solution since we must consider the medical procedures and comfort of the patients who undergo the insertion of the seeds. Considering the smallest possible projection of a seed inside the portal image, the size of the seed in pixels is comparable to the spatial frequency of the noise in the image. It is therefore not straightforward to apply a smoothing to the image before detecting the seeds, because it is possible that the seed will be erased from the image (smoothing out the seed pixels into background pixels). The possibility of removing the seed in the smoothing process, results in an algorithm that is less robust, making it unacceptable for clinical practice.

The algorithm presented in this chapter makes use of a priori known geometrical information. The location of the prostate at treatment planning time is calculated, therefore we know approximately where the seeds are in the portal image before treatment. We also know the seed shape and size, as well as the spatial resolution of the imaging system used during treatment, which gives us the size of a seed in terms of the number of pixels in the portal image. This knowledge results in a small search area to look for a seed with the minimum and maximum number of pixels a projected seed can have in the portal image.

\subsection{Preliminaries}

\subsubsection{The Region Of Interest}

The only a priory information available is the location of the seeds in the prostate at the time of simulation. The BEV 2D-projection of each field is reconstructed from CT data giving the seed positions. This knowledge speeds up the process by providing an approximate area in the image to start the search for a seed, which we refer to as 
the Region of Interest (ROI). In the portal image, the center of the ROI is placed at a previously known seed location computed from the reconstructed BEV.

The prostate has a tendency of moving within the pelvis, predominantly in the cranial-caudal direction, as verified in the work $[3,11,13,46]$. If we consider a maximum shift in position of $10 \mathrm{~mm}$ in this direction (y: head to toe) and $5 \mathrm{~mm}$ in the medial-lateral direction ( $\mathrm{x}$ : hip to hip), the ROI is a rectangle. At the ORCC, years of clinical experience suggest that a prostate shift of $10 \mathrm{~mm}$ is an extreme case, therefore we can be confident that if the ROI is properly placed in the portal image, the seed will be contained within this region. Consequently, the ROI has a height of $20 \mathrm{~mm}$ and a width of $10 \mathrm{~mm}$ corresponding to the expected amount of prostate movement in each axis. Figure 5.2 demonstrates the size of the small area covered by the ROI with respect to the portal image size.

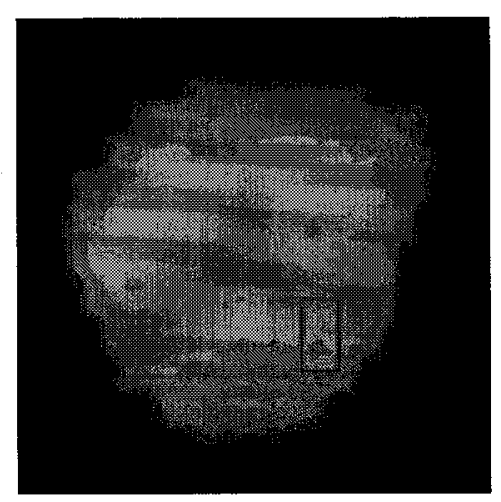

Figure 5.2: Region of Interest (ROI) over seed 3.

\subsubsection{The Seed Geometry}

An important consideration in the search for a gold seed in a portal image is the seed size and shape. With this information, we can calculate the minimum $P_{\min }$ and the maximum $P_{\max }$ number of pixels a projected seed has in the portal image. The standard seed shape used at most regional cancer centers is a cylinder. The smallest projection of a seed in a portal image then consists of a circle, the base of the cylinder, and the largest projection consists of a rectangle, the side view of the cylinder. 
To detect a seed accurately, the seed's intensity values must be examined with the aim of finding an appropriate threshold that will differentiate the seed pixel values from the background. However, the intensity ranges for gold seeds differs for each seed and also differs from image to image. Therefore, to detect the seeds, a global thresholding method is not feasible and an adaptive technique must be employed.

\subsection{Algorithm Steps}

The crux of the algorithm is to develop this adaptive technique in order to determine seed ranges for each image. Once a range is established for a seed, the seed shape is defined and the center of gravity (CoG) of the seed is calculated. The CoG is calculated with the contour points of the detected seed shape and the location of a seed in an image is given by its CoG. Preprocessing steps such as smoothing and enhancing are not employed to obtain the CoG of a seed more accurately. The main reason for this is that these types of preprocessing steps tend to distort the original image and hence, can introduce more error with respect to seed position. We will show this phenomenon experimentally.

Figure 5.3 displays four reconstructed images of 1) an original seed in a portal image, the results after 2) a smoothing, 3) a contrast enhancement method is applied and 4) the ideal case where the detected seed has the same geometry as the original seed image in 1). The red square represents the CoG for each seed geometry. In this case, the smoothing process changed the shape of the seed but the seed has the same CoG as the original image. Conversely, the contrast enhanced image has neither the same shape nor the same CoG as the original projected seed image.

Ideally, we want to obtain a seed geometry which has a one-to-one correspondence with the geometry of the original seed projection in terms of the number of pixels and orientation. Since accuracy is paramount, we work with the original images of the seed. Table 5.1 gives an outline of the four basic steps of the algorithm (on page $36)$. 

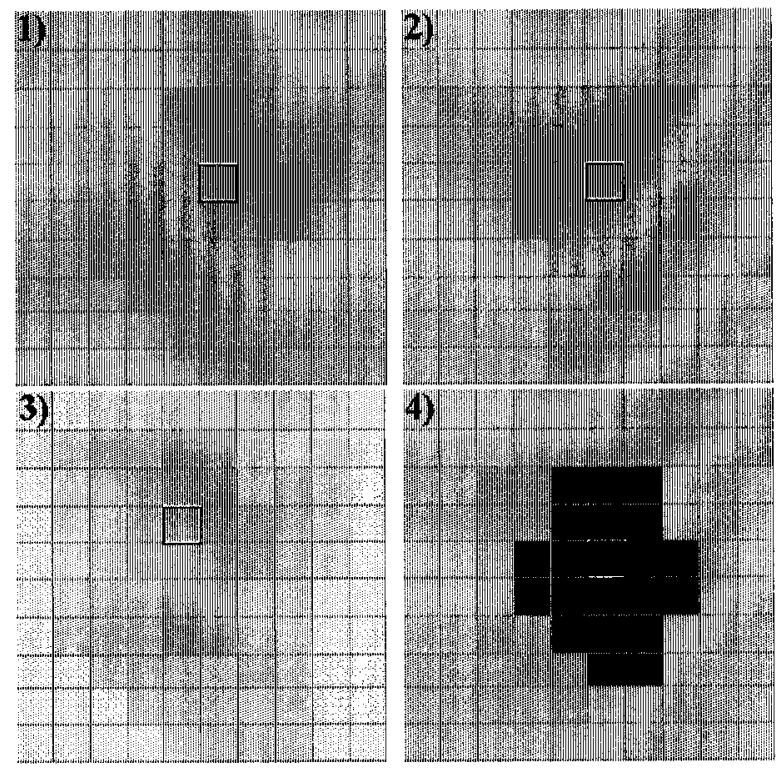

Figure 5.3: Reconstructed seed images: 1) original 2) smoothed 3) contrast enhanced and 4) ideal detection.

\subsubsection{Seed Intensity Ranges}

The algorithm starts by analyzing the pixel intensities in the ROI through a set of consecutive non-intersecting $3 \times 3$ blocks of pixels. The standard deviation of each block is calculated. In portal images, a seed is darker than its surrounding neighbouring pixels. If one of the blocks cuts a seed (seed edge is inside the block), it will contain seed values and homogeneous background values, if not, it will consist either of homogeneous background values or of only seed values. The standard deviation is calculated from the intensity histogram of a block. Where the standard deviation is at a maximum, the spread of values in the histogram of the $3 \times 3$ block is wide covering a broader range of greyscale values. The block with highest standard deviation is chosen as the first candidate block to possibly contain seed pixels, providing an area within the ROI to start the search for a seed.

Once a location to start the search inside the ROI is determined, the procedures followed in steps 2 and 3 in Table 5.1 are presented in the flow chart in Figure 5.4. For each location determined by the location of the block with highest standard deviation, these steps are applied in the order presented in the flow chart. 
For each seed:

Step 1: Locate the ROI $R$ in the portal image by centering it at the previously known seed location $s_{p}$. Locate the $3 \times 3$ block with highest standard deviation.

Step 2: Find an intensity range for the new seed location $s_{n}$. Keep track of the number of pixels $x$ contained in the current range $r$. If $\mathrm{x}<P_{\text {min }}, r++$ and grab all pixels having the corresponding intensity. If $\mathrm{x}>P_{\text {max }}, r$ - and remove all pixels having the corresponding intensity and the search is stopped (detected set of seed pixels).

Step 3: Verify the geometry of seed $s$ and calculate the $\mathrm{CoG} c_{s}$ of the resulting polygon.

For the three seeds in the pair images:

Step 4: Calculate the Euclidean distance between the three seeds in each image and compare with the distances between seeds calculated at treatment planning.

*If a seed detection failure occurred, go to Table 5.2

Table 5.1: Steps of Algorithm.

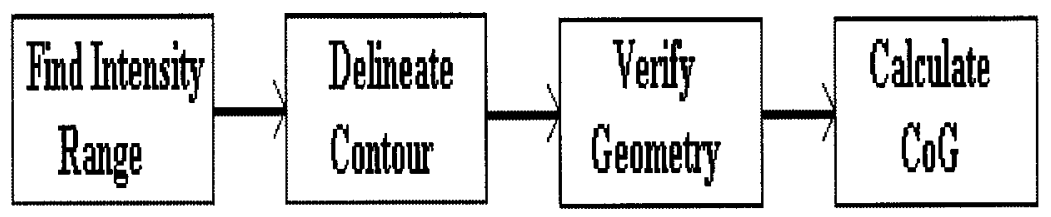

Figure 5.4: Flow Chart of algorithm steps to detect one seed.

The next step involves finding a seed intensity range, starting with the lowest pixel value (darkest pixel) in the first candidate block (block with highest standard deviation). The four neighbouring blocks bordering with three pixels (above, left, below and right blocks) and the four neighbouring blocks bordering with one diagonal pixel (adjacent diagonal blocks) are examined for intensities. The number of pixels that have this intensity is counted. If the number of available pixels is less than $P_{\text {min }}$, the current seed range is increased by one intensity value and all pixels in the current and neighbouring blocks with that value are added in the set of potential seed pixels. This process is repeated for each new neighbouring block within the ROI containing seed values within the current range. 
If the number is greater than $P_{m a x}$, the seed range is decreased by one intensity value and pixels having that value are removed from the set of candidate seed pixels. The search stops here and the seed's intensity range is the range resulting from removing the last investigated intensity that made the number of candidate seed pixels be greater than $P_{\max }$. The first block with highest standard deviation may not contain seed pixels but its neighbouring blocks may. Therefore, the bordering blocks may contain lower intensities, if so, there is a new minimum value to restart the search with. Figures 6.3 gives an example where the first $3 \times 3$ block with highest standard deviation (boxed in region) did not contain seed pixels (blue intensities) but the neighbouring block above contained a new minimum value of 155 which is included in the set of seed pixels. This neighbouring block is now treated as the first $3 \times 3$ block with highest standard deviation, the search starts within this new block with the new minimum.

If the first candidate block is at or very close to the ROI edge (cannot look at all of eight neighbours), the ROI border may be intersecting the seed we are trying to detect. If this occurs, the ROI is moved by 9 pixels (3 blocks) horizontal/vertically if the ROI intersects the block at its edges, or diagonally if the ROI intersects the block at one of its corners.

An iteration consists of looking at the neighbours of a new block chosen as having pixel values within the current intensity range. Iteration 1 consists of looking at the first chosen block with highest standard deviation along with its neighbours. Iteration 2 consists of looking at one of the neighbouring block's neighbours. The number of iterations it takes the algorithm to converge is the number of blocks inside the ROI whose neighbours were looked at to find seed pixels. The search stops when all surrounding blocks bordering the candidate blocks are discarded as having too high intensities and the number of pixels having values included in the seed range is within $P_{\min }$ and $P_{\max }$.

Figure 5.5 displays the intensity values for two seeds. In the first example, the intensity range contains only 2 intensities. The algorithm starts with the minimum value (6 pixels with value 168) and grows until the number of candidate seed pixels is greater than $P_{\max }$. In this case, when looking at value 170 (blue pixels), the number of 
pixels in the candidate set jumped to 32 so that the threshold value in differentiating the seed is set to 169. In other words, using the knowledge on the seed size and the spatial resolution of the imaging system, a seed will never project to such a big set of pixels, therefore the pixels with value 170 are considered to be background pixels. However, in the second example, four intensity values were needed to cover the seed and here the threshold value is chosen to be 170 . The different colours in the images correspond to the set of pixels added in the set of candidate seed pixels when a new intensity is added to the current intensity range $r$ and, the blue pixels correspond to the last iteration before converging.

\begin{tabular}{|c|c|}
\hline 171169170170170171 & \\
\hline $\begin{array}{llllll}169 & 168169169170 & 171\end{array}$ & 173172172172172 \\
\hline 171170168168169170 & 172172170171172172 \\
\hline 170169168169170171 & 172171168170171173 \\
\hline 170170169169170171 & 172171167169172174 \\
\hline & $59170 \quad 171172$ \\
\hline 72172171171171171 & 171170171172 \\
\hline
\end{tabular}

Figure 5.5: Intensity ranges for two seeds.

Once a range of intensities is created, the corresponding set of pixels must be connected, forming an irregular polygon as seen in figure 5.6.

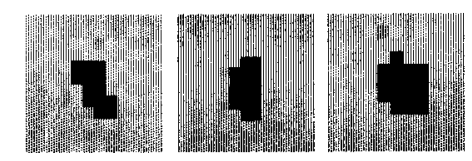

Figure 5.6: Seed shape, size and orientation.

The purpose in finding a set of candidate seed pixels, is to delineate the seed contour such that the CoG of the polygon will be as close as possible to the true $\mathrm{CoG}$ of the seed. A contour tracing algorithm is employed to obtain the contour points of the polygon created from the set of candidate seed pixels. The boundary of the set of pixels is extracted with the Moore-Tracing algorithm, a method used to contour square tessellations ${ }^{1}$ that are 8 -connected [45].

\footnotetext{
${ }^{1} \mathrm{~A}$ mosaic pattern.
} 


\subsubsection{Geometry Verification}

The images used in this thesis have been captured with Beamview ${ }^{\text {plus }}$ from Siemens Oncology Care Systems at the University of California San Francisco (UCSF) Medical Center. The seed size is $1.6 \times 3 \mathrm{~mm}$ at isocenter and the spatial resolution at isocenter of their imaging system is $0.55 \mathrm{~mm}$ per pixel in both the $\mathrm{x}$ and $\mathrm{y}$ directions. As seen in Figure 5.7, the seeds are projected onto the imaging plate (EPID plane) at $100 \mathrm{~cm}$ from the source. The ROI dimension in the image is then $18 \times 36$ pixels (corresponding to $10 \times 20 \mathrm{~mm}$ ), a small search area.

The dimensions of the seed is approximately 3 pixels $\mathrm{x} 5$ pixels. The seed size in terms of area may then vary from 5 pixels (circular projection) to at most 16 pixels (rectangular projection). A perfect side view projection of the seed would give a set of 15 pixels but taking noise into consideration, the threshold is set to 16 pixels.

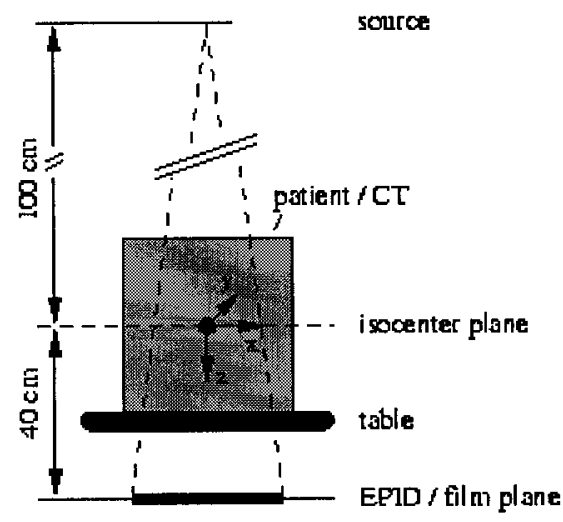

Figure 5.7: Seed Projection from the isocenter plane.

The set of candidate pixels must be connected. If the set of contour points do not contain all candidate seed pixels, then the set consists of disconnected pieces. If a piece contains less than $P_{\min }$ pixels, the piece is removed from the set. Otherwise, the largest piece in terms of the number of pixels is taken as the first possible seed. All remaining pieces having less than or equal than $P_{\max }$ pixels are kept for future reference in case the current chosen set of pixels is discarded in the following verification steps. If there are pieces of equal size, an arbitrary piece is chosen and the process continues. 
Noise is a factor to consider when the images are generated with EPIDs. In the process of capturing a portal image, a pixel's value may be changed to a darker or lighter intensity. Once we have a set of connected pixels to work with, there exists the possibility that some pixels have been changed to a lighter intensity, creating a hole inside the set of pixels forming the polygon. For instance, if the seed in the portal image is the projection of the base of the cylinder, the set of pixels to detect will be very small. If one pixel is changed, we do not want to reject this set of pixels. Figure 5.8 gives an example of a set of candidate seed pixels which may possibly be the projection of the base of the cylinder. In this case, the hole pixel is added to the set of candidate seed pixels. Considering a larger set of seed pixels, if one pixel is changed, one of its neighbours may be influenced creating a hole of 2 pixels. Consequently, if a hole is detected inside the polygon, the hole must consists of at most 2 pixels for the polygon to be considered a possible seed. If the hole contains a larger set of pixels, the polygon is rejected.

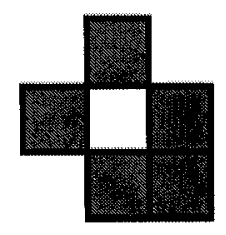

Figure 5.8: Detected Polygon with a one pixel hole.

For the area of the polygon to be accepted, the claims below must be true.

1) The smallest seed geometry must fit inside the detected polygon.

2) The polygonal shape must be "blob like".

The smallest seed outline is a circle with a diameter of 3 pixels. This implies that for any projection of a seed, independent of the orientation, a width of 3 consecutive pixels will exist in at least two directions. Moreover, the two directional lines of 3 consecutive pixels must cross at the middle pixel as seen in Figure 5.9, representing the diameters of the circle. Other possible diameter projections are obtained by rotations and/or reflections of case 3). Consequently, to verify the geometry of a detected seed, the width of the polygon delineating the seed is checked in the horizontal, vertical and diagonal directions. If none of the patterns in Figure 5.9 are detected in the 
polygon, the candidate seed is rejected.
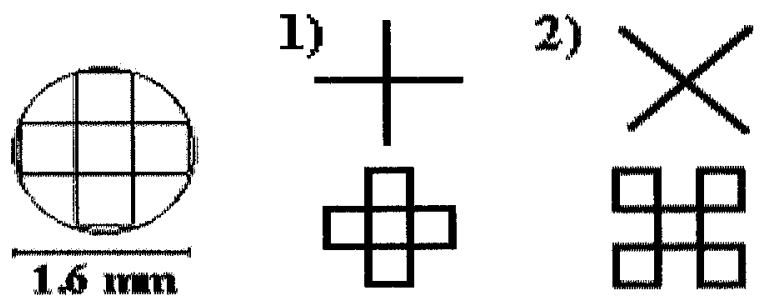

3)

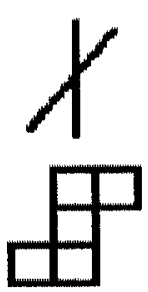

Figure 5.9: Possible circle projections.

Verifying that the smallest seed projection fits inside the detected polygon is necessary but not sufficient. Claim 1) alone does not guarantee that the detected shape is indeed a possible seed. As a result, a final geometry verification step is carried out. The area created by the set of seed pixels is examined to validate if the polygonal area has a seed's geometry. The projection of a seed in the image should have a shape between a circle and a rectangle. In other words, the polygon must have a "blob like" shape and not a "line like" shape as seen in Figure 5.10.

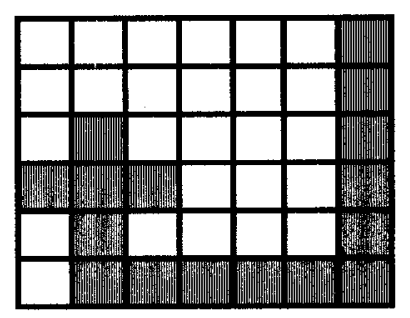

Figure 5.10: Rejected seed geometry.

In attempt to verify claim 2), different methods were investigated. One method calculates the percentage of the area the polygon covers within the bounding box of the polygon. Unfortunately this method is not adequate. Case A) in Figure 5.11, the detected polygon should be accepted as a "blob like" shape but covers only $40 \%$ of the bounding box. On the other hand, in case B) the shape is "line like" and the polygonal area covers over $60 \%$ of the area in the bounding box. Therefore, no threshold value in accepting a seed shape with this property is feasible. 
Another possible solution is to count the number of single pixels within the boundary of the polygon. Single pixels are considered to have only one neighbour within the polygon. Considering case B), there are 10/12 single pixels and it clearly should not be accepted as a possible seed but in case C), there are 6/9 single pixels and the geometry should be accepted.
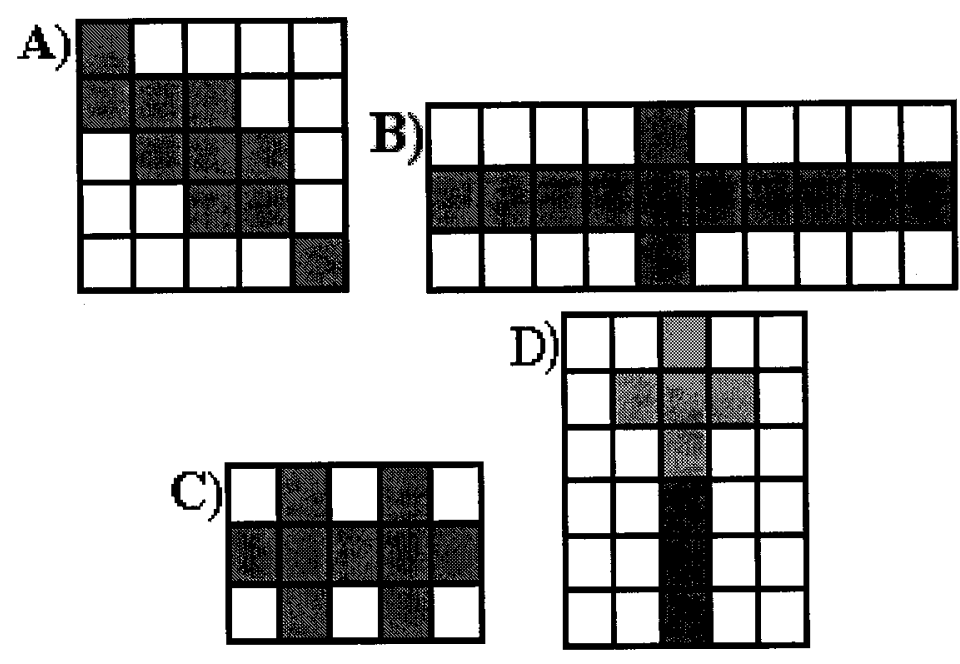

Figure 5.11: Seed geometry: acceptable in A) and C), not acceptable in B) and D).

Therefore, the final decision involves finding the "line like" portions of the polygon and calculating their lengths in pixels. If the portion does not cover the majority of the area of the polygon, the geometry of the detected polygon is accepted as having a "blob like" shape and the process proceeds to calculate the CoG. This part of the verification process is only applicable to detected seeds having more than 7 pixels. The reason is that if you look at the smallest seed projections in Figure 5.9, pattern 3) consists of a line of 5 pixels and this shape is accepted. Letting at most two pixels append at the end pixels, the seed shape should still be accepted. Case D) in Figure 5.11, there are 8 seed pixels and the "line like" portion covers 3 pixels (darkest pixels) of the polygon. Using this example, the threshold of acceptable "line like" portions is set to $30 \%$ such that if the portion covers less than $30 \%$ of the polygonal area, the seed geometry is accepted and the CoG is calculated. 


\subsubsection{Center Of Gravity}

To calculate the CoG of a polygon, the polygon must be partitioned where each partition is weighted with respect to the area it covers in the polygon [51]. The CoG of each partition is calculated and the CoG of the polygon is the weighted sum of the partitions CoGs. The set of candidate seed pixels comprises of rectilinear polygons since all edges are either vertical or horizontal. Therefore, decomposing rectilinear polygons into convex quadrilaterals was investigated $[22,42]$. However, since the polygons have at most 16 pixels, using a triangulation method that is not the most, efficient but simple to implement is permissible. For that reason, the Graham Scan algorithm is employed [17]. Once the polygon is triangulated, the $\mathrm{CoG}$ and the area of each triangle is calculated. The area of the polygon is the sum of the triangular areas. The ratio of each triangular area with respect to the polygonal area are taken and multiplied with the corresponding CoGs of each triangle. The sum of these products returns the location of the seed. Figure 5.12 displays an original portal image, the related image with the three seeds detected and thirdly, the image with the corresponding CoGs circled in red.
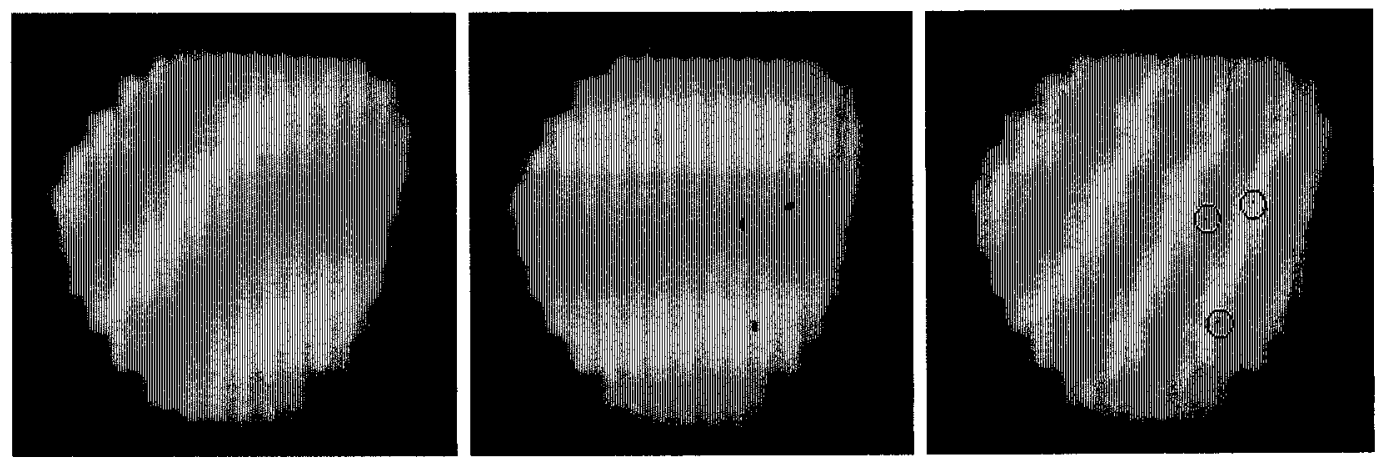

Figure 5.12: Original image, image with detected seeds and image with CoGs.

\subsubsection{Intra Seed Distances}

The three seeds are detected in a pair of orthogonal images taken at perpendicular field projections. Once the three seeds are detected in both images, the seed locations are independently verified by comparing the 3D-Euclidean distance between the seeds 
in image pairs to the distances between the seeds in the corresponding fields reconstructed from treatment planning. Figure 5.13 demonstrates a 2D view of distances between seeds in a LAO and RAO image pair.
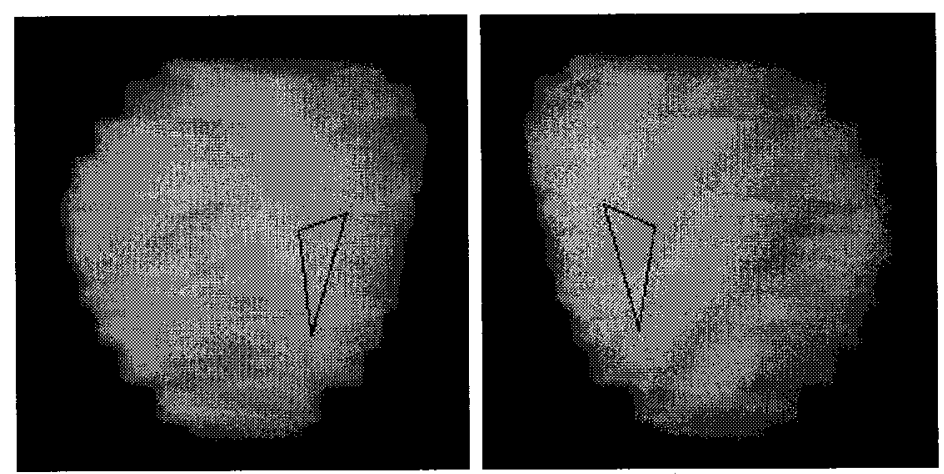

Figure 5.13: LAO and RAO with intra-seed distances.

If the differences are less than a threshold determined by the ORCC, the detected seeds are accepted. Otherwise, either the detection algorithm failed, the seeds migrated within the prostate or the prostate gland changed shape. If the seeds migrated, the patients can undergo a re-insertion of the seeds inside the prostate and continue the treatment. If the three seed distance ratios are comparable to the ratios obtained from CT during treatment planning, the patient may still be treated with the method presented in this thesis. However, if the seeds are undetectable the patient is not a candidate for the image guided technique.

\subsection{Seed Detection Failure}

The algorithm presented above fails when a detected shape with proper geometry is in fact not a seed or if no seed is found in the ROI. Table 5.2 gives an outline of major steps carried out after a seed detection fails.

If a seed shape is rejected based on the intra-seed distances, the first step consists of checking if there are other pieces that have been detected. If other pieces were detected and the geometry verification process accepts the shape, recalculate the intra-seed distances in corresponding image pairs with this set of pixels. If no other 


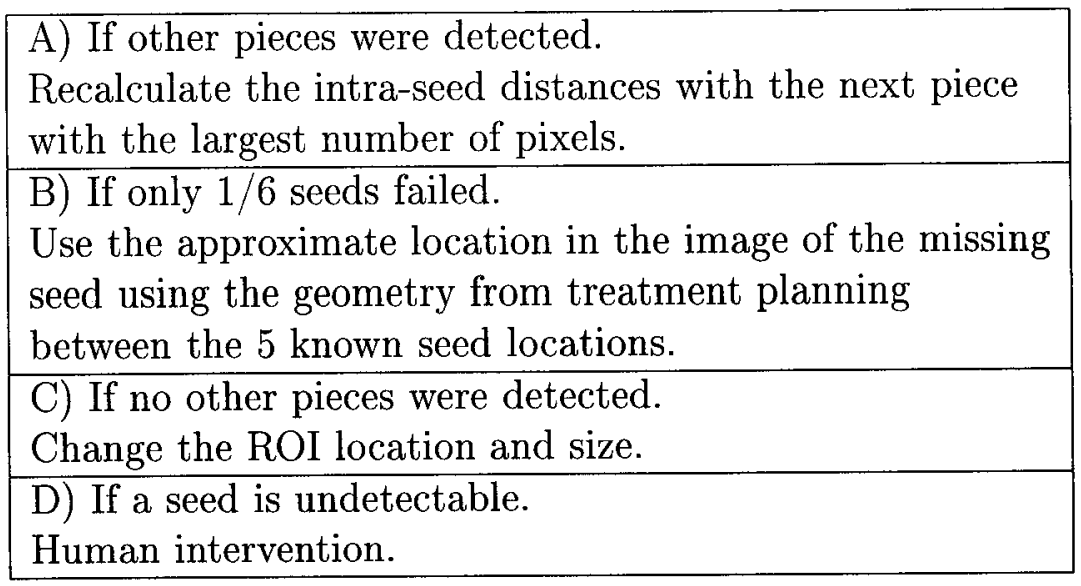

Table 5.2: Steps for Seed Detection Failure.

pieces exist, the geometry between the three seeds can be helpful if only one of the seeds in the image pair is missing. Using the location of the missing seed in the image where all three seeds are correctly detected, we can focus on the corresponding location in the image where the seed is missing. If the seed is still rejected and no other pieces exist, the ROI coordinates are changed.

There are two possible cases where a detection failure may occur. Firstly, if a seed projection contains less than 9 pixels, it is possible that none of the $3 \times 3$ blocks cut the seed. The closest $3 \times 3$ block may completely overlap the seed such that no significant standard deviation is detectable at that location inside the ROI. Secondly, the ROI may simply not contain a seed.

Depending on the reason of failure explained above, either we want a $3 \times 3$ block to cut the missing seed to get a noticeable highest standard deviation, or the ROI to contain the seed. To ensure the first case, the ROI is moved by one pixel in a diagonal direction, as long as it does not move towards a field edge. To correct the second case, the ROI size is increased in dimension by one block on each side of the ROI, increasing the search area in width and height by six pixels.

If all of the above procedures fail, the search is stopped and a human observer intervenes. 


\section{Chapter 6}

\section{Implementation and Experiments}

Based on experiments carried out on real patient images, the algorithm is fast, accurate and simple. In previous results $[31,32]$ every pixel of the input image is processed at least once. The algorithm presented in this thesis avoids full image processing by taking advantage of the ROI. A standard portal image size is $512 \times 480$ pixels, and one ROI as explained in the previous chapter covers only $0.26 \%$ of the total image, yielding approximately 400 times improvement in performance. Furthermore, in comparison to other work [37], the performance of the algorithm is improved by removing all preprocessing steps. Smoothing and contrast enhancement can introduce error and an unaltered seed shape is important to accurately find the CoG of a projected seed.

\subsection{Test Cases}

Different test cases were applied: $(i)$ the ROI contains a seed, $(i i)$ the ROI contains no seed, (iii) the ROI boundary intersects a seed, and (iv) the ROI is placed very close to the edge of the radiation field. Figure 6.1 is an example of a portal image where seeds 2 and 3 in the image are very close to the field edge. For visualization purposes, the contrast image contains the three seeds circled in black. 

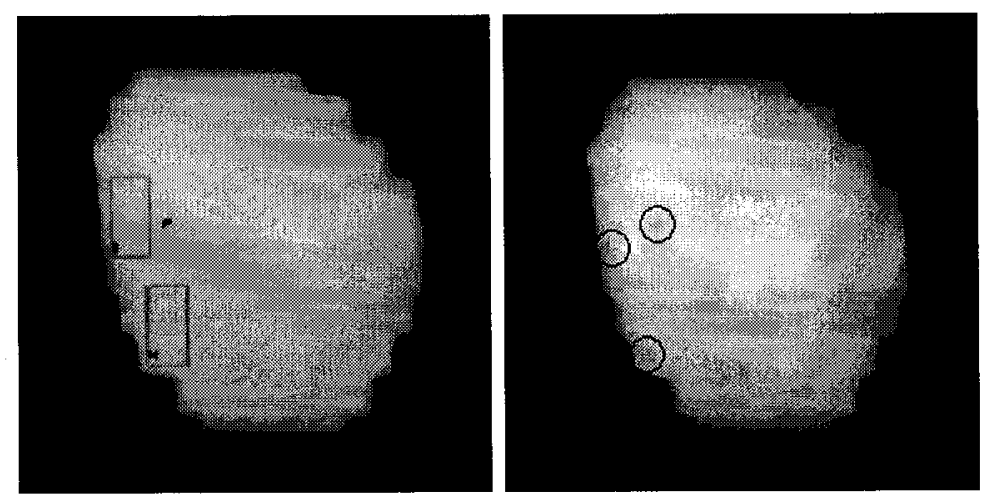

Figure 6.1: Image with ROIs placed close to the field edge and the corresponding contrast enhanced image.

\subsection{Results}

The seed detection algorithm is highly accurate. In all cases considered, the seeds are detected at their correct location. This is true also for low intensity differences between seed values and background values (e.g., seed value is 177 and the background value is 179$)$.

In case $(i)$ - the ROI contains a seed- the algorithm converges rapidly to a proper seed size and shape. The results show that when the ROI contains a seed, from the first candidate block with highest standard deviation, the algorithm finds an appropriate seed range within a reasonable small number of iterations in looking at neighbouring blocks. Figure 6.2 contains a graph where the horizontal axis shows the block number in the ROI and the vertical axis displays the corresponding difference between the lowest and highest pixel values in the histogram. The set of $3 \times 3$ blocks containing seed pixels is to the right of the graph and the boxed in $3 \times 3$ block is the first chosen block with highest standard deviation. In this case, the first candidate block contains seed values (blue pixel values) therefore, seed values are found in the first iteration. In Figure 6.3, seed values are found in the second iteration since the seed values are above the first candidate block (a new minimum value is found above). Table 6.1 contains results where the first column gives the seed number (18 seeds in 6 images), the second column shows whether or not the first candidate block with highest standard deviation contained seed pixels. The third column contains 


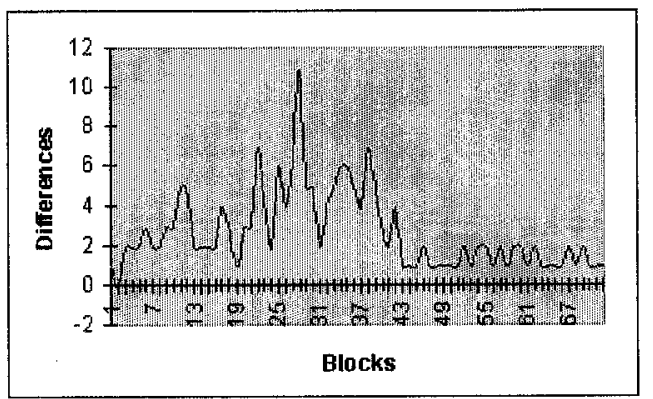

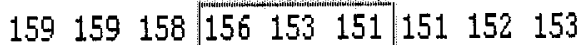

\begin{tabular}{lll|lll|lll}
158 & 156 & 156 & 152 & 147 & 146 & $\mathbf{1 4 9}$ & 152 & 153
\end{tabular}

\begin{tabular}{lllllll|lll}
156 & 154 & 153 & 149 & 146 & 145 & 149 & 152 & 154
\end{tabular}

$\begin{array}{lllllllll}154 & 153 & 149 & 147 & 145 & 145 & 149 & 153 & 154\end{array}$

$\begin{array}{lllllllll}154 & 153 & 149 & 147 & 146 & 147 & 150 & 153 & 155\end{array}$

$\begin{array}{llllllllll}154 & 154 & 152 & 151 & 150 & 150 & 151 & 153 & 154\end{array}$

Figure 6.2: Detected seed values on iteration 1.

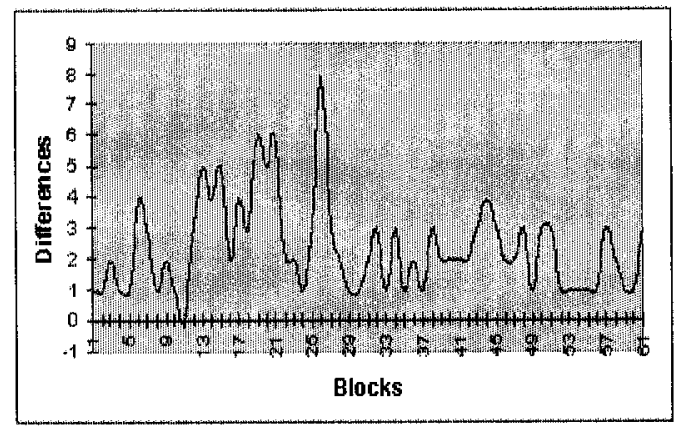
$\begin{array}{lll}159 & 156 & 157\end{array}$
158156156
157155156
157155156
$\begin{array}{llll}158 & 156 & 157\end{array}$
$160 \quad 158 \quad 158$
$\begin{array}{lll}163 & 160 & 160\end{array}$
$164 \quad 162 \quad 161$
$\begin{array}{lll}168 & 167 & 166\end{array}$

Figure 6.3: Detected seed values on iteration 2.

the number of pixels the detected seed has. The fourth column gives the number of iterations it took for the algorithm to converge to a seed geometry and finally, the detected seed intensity range is given. In over $60 \%$ of the cases $(12 / 18 \sqrt{ })$ the seed was immediately detected. Notice that for seeds 1,2 and 3 the seed ranges do not overlap and with seeds 10,11 and 12, the seed range sizes are considerably different. Seed 10 contains only four intensity values in the range, however seed 11 contains thirteen intensity values. These results confirm the assertion that seed intensity ranges within a portal image can differ significantly between seeds.

The number of iterations is bounded by the number of blocks containing seed pixels. Figure 6.4 gives two example seeds which correspond to seeds 7 and 11 in Table 6.1. The seed on the left is overlapped by six different blocks so that it took 7 iterations to converge and the range contains only two intensities. On the other hand, the example on the right corresponding to seed 11 took only 3 iterations to converge since there are only two blocks overlapping the seed, and the intensity range contains 


\begin{tabular}{|c|c|r|c|c|}
\hline Seed & $\begin{array}{c}\text { First } \\
\text { Block }\end{array}$ & Pixels & $\begin{array}{c}\text { Itera- } \\
\text { tions }\end{array}$ & $\begin{array}{c}\text { Seed } \\
\text { Range }\end{array}$ \\
\hline 1 & $\sqrt{ }$ & 16 & 5 & $145-149$ \\
2 & & 16 & 3 & $155-158$ \\
3 & $\sqrt{ }$ & 12 & 4 & $138-142$ \\
\hline 4 & $\sqrt{ }$ & 16 & 5 & $166-169$ \\
5 & & 11 & 5 & $160-163$ \\
6 & $\sqrt{ }$ & 11 & 4 & $159-161$ \\
\hline 7 & & 16 & 7 & $168-169$ \\
8 & $\sqrt{ }$ & 11 & 4 & $161-163$ \\
9 & $\sqrt{ }$ & 14 & 4 & $155-157$ \\
\hline 10 & $\sqrt{ }$ & 13 & 5 & $149-152$ \\
11 & & 15 & 3 & $122-134$ \\
12 & & 14 & 5 & $132-136$ \\
\hline 13 & $\sqrt{ }$ & 14 & 4 & $171-174$ \\
14 & $\sqrt{ }$ & 13 & 5 & $177-178$ \\
15 & & 14 & 3 & $173-174$ \\
\hline 16 & $\sqrt{ }$ & 12 & 4 & $176-180$ \\
17 & $\sqrt{ }$ & 9 & 4 & $167-170$ \\
18 & $\sqrt{ }$ & 12 & 3 & $167-171$ \\
\hline
\end{tabular}

Table 6.1: Results with test case $(i)$ - ROI overlapping a seed.

thirteen intensities. Therefore, the number of steps it takes the algorithm to converge is independent on the number of intensities a seed contains.

In case $(i i)$ - the ROI contains no seed - one case out of five, the detected seed geometry is accepted. Table 6.2 contains five cases where the ROI overlapped different homogeneous background regions. The first column gives the region number, the second columns gives the number of pixels the seed geometry contains, followed by the number of iterations the algorithm took to converge, the seed intensity range is given in the fourth column, column five gives the number of detected pieces and finally, whether or not the detected geometry is accepted. In the first case, with a large number of iterations, a seed shape is recognized as having the proper geometry. In the remaining cases, the seeds are rejected by geometric computations; either the number of pixels is too small or the smallest seed projection does not fit inside the detected polygon. 


\begin{tabular}{|c|c|}
\hline \begin{tabular}{lll|llllll}
172 & 172 & 171 & 171 & 171 & 171 \\
\end{tabular} & \\
\hline $171 \quad 169170 \mid 170170171$ & \\
\hline $\begin{array}{llllllllll}169 & 168 & 169 & 169 & 170 & 171\end{array}$ & \\
\hline 171170168168169170 & 134135136 \\
\hline $171170168 \mid 168169170$ & 130133134 \\
\hline $\begin{array}{llllllllll}170 & 169 & 168 & 169 & 170 & 171\end{array}$ & 127131133 \\
\hline $\begin{array}{llllll}170 & 170 & 169 & 169 & 170 & 171\end{array}$ & 127131131 \\
\hline $171171170 \mid 170170171$ & $125 \quad 130133$ \\
\hline \begin{tabular}{llll|ll|}
172 & 172 & 171 & 171 & 171 & 17
\end{tabular} & 122129136 \\
\hline
\end{tabular}

Figure 6.4: $3 \times 3$ blocks overlapping different seeds.

\begin{tabular}{|c|c|c|c|c|c|}
\hline Region & Pixels & $\begin{array}{c}\text { Itera- } \\
\text { tions }\end{array}$ & $\begin{array}{c}\text { Seed } \\
\text { Range }\end{array}$ & Pieces & Geometry \\
\hline 1 & 9 & 9 & $134-135$ & 1 & $\sqrt{ }$ \\
\hline 2 & 12 & 4 & $156-160$ & 2 & \\
\hline 3 & 10 & 3 & 163 & 2 & \\
\hline 4 & 4 & 7 & 160 & 1 & \\
\hline 5 & 4 & 4 & $148-149$ & 1 & \\
\hline
\end{tabular}

Table 6.2: Results with test case (ii) - ROI overlapping a background region.

In case ( $i i i)$ - the ROI intersects a seed - the ROI was placed to cut the seed at two locations of the ROI's boundary; at the edges and at the corners. The seeds are discovered such that the maximum standard deviation is at, or close to, the set of seed pixels. Given that the ROI intersects the seed, the search is interrupted when looking for further possible seed pixels. Consequently, the ROI is moved to completely overlap the candidate region exposing the rest of seed pixels.

In the last case, case $(i v)$ - the ROI placed close to a field edge - the automatic detection fails because manual intervention is necessary to ensure that the ROI is at a certain distance from the edge. The reason is that since the background in the portal image is black and the foreground is light grey, the pixel intensities degrade significantly and rapidly towards the field edge, causing the standard deviation to maximize not at a seed location. The algorithm starts with the lowest intensity value of a block, if the block is close to the field edge, new minimum values are found when searching towards the edge. The graph in Figure 6.5 displays the intensity values of 
the horizontal line in the original portal image. The example demonstrates that in the portal image, the pixel values decline rapidly from the field region to the background region. In this example, the field edge is at pixel 41 .
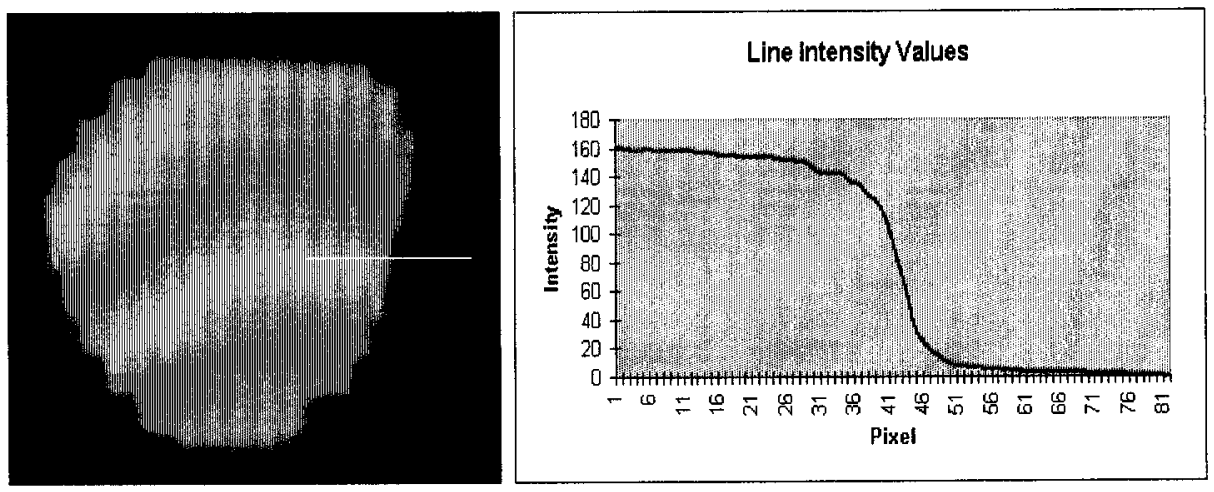

Figure 6.5: Edge pixel intensity graph of the corresponding horizontal line in the portal image.

Figure 6.6 gives an example of the case where there are two blocks with the same histogram width. The block with highest standard deviation is at the far right of the graph circled in red. When the ROI is moved by two pixels away from the field edge, the "extra" maximum standard deviation is removed resulting in the second graph where now the block with highest standard deviation results in a proper seed detection.
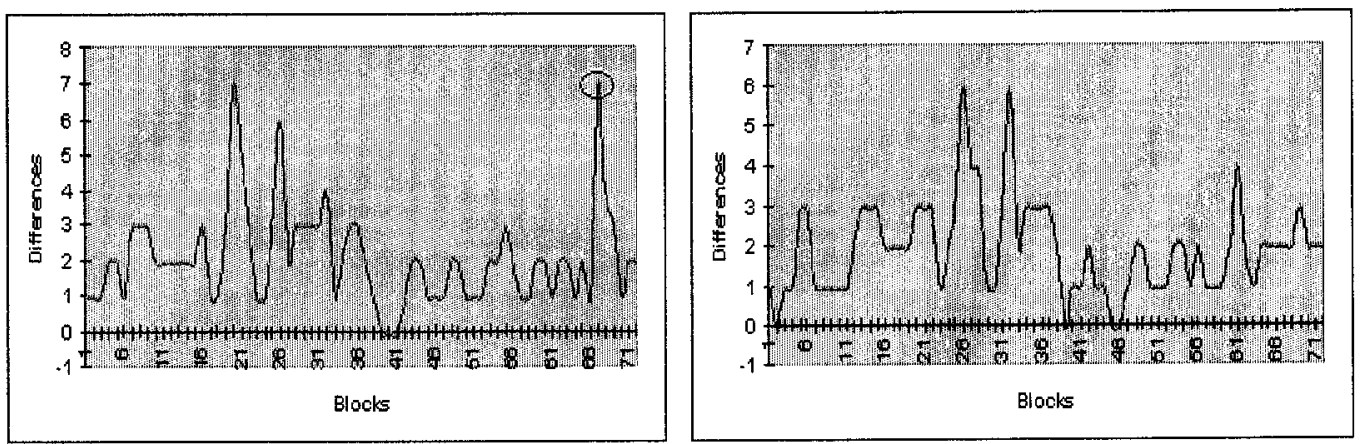

Figure 6.6: ROI values placed close to a field edge. 


\subsection{Additional Testing}

A comparison was made between the detected $\mathrm{CoG}$ coordinates in the un-enhanced images with the CoGs in corresponding smoothed images. The spatial filters used to smooth the images are the mean and median filters with $3 \times 3$ kernels. In 16/18 seeds (Table 6.1), the CoGs differ in at most 1 pixel location, which corresponds to a difference of at most $0.55 \mathrm{~mm}$. For the remaining two seeds, the detection failed: the geometry of seed 15 failed in both smoothed images and seed 17 failed in the median smoothed image. Table 6.3 contains the three seeds with the number of pixels the detected seed has along with the intensity range when first detected, and the last column shows weather or not the detected polygon is indeed a seed.

\begin{tabular}{|l|c|c|c|}
\hline \multicolumn{1}{|c|}{ Seed } & Pixels & $\begin{array}{c}\text { Seed } \\
\text { Range }\end{array}$ & $\begin{array}{c}\text { Seed } \\
\text { Location }\end{array}$ \\
\hline \hline 14-mean & 5 & 173 & $\sqrt{ }$ \\
\hline 14-median & 4 & $173-174$ & \\
\hline 17-median & 6 & $167-168$ & $\checkmark$ \\
\hline
\end{tabular}

Table 6.3: Seed detection failures in smoothed images.

Comparing with the corresponding values in Table 6.1, the number of pixels and intensity ranges the detected seeds have are significantly smaller. Smoothing replaces pixel values with the mean or median of the surrounding pixel values, which in this case, decreased the border around the detected polygons. For this reason, seeds 14mean and 17-median, although are correctly located at a seed and have the same CoGs as in the un-processed images, are rejected because the smallest seed projection does not fit inside the polygon, as seen in Figure 6.7. If the seed projected in an image is the smallest projection so that before smoothing, the polygon contains 5 pixels, it is highly probable that after a smoothing filter is applied, the projection of the seed is replaced by surrounding pixels (background pixels) completely erasing the seed.

One final test is carried out to see what happens when the algorithm is used to detect seeds in a lateral field image. The difficulty lies in detecting seeds that are close the bony structures in the portal image. With respect to the number of pixels of the bones, a seed is exceedingly small. Figure 6.8 gives an example of a lateral 


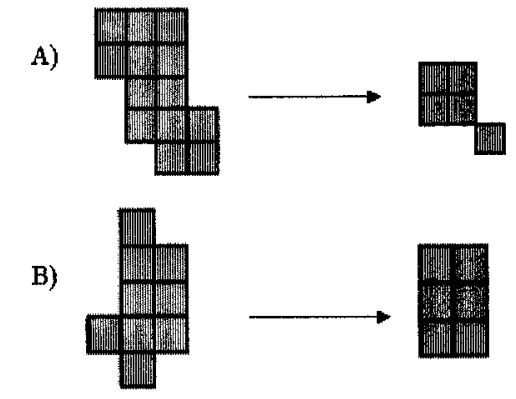

Figure 6.7: Original seeds with corresponding smoothed seed.

image with the related contrast enhanced image where the bones are clearly visible. The yellow circle shows the problem area in the image; seed 3 next to the hip bone.
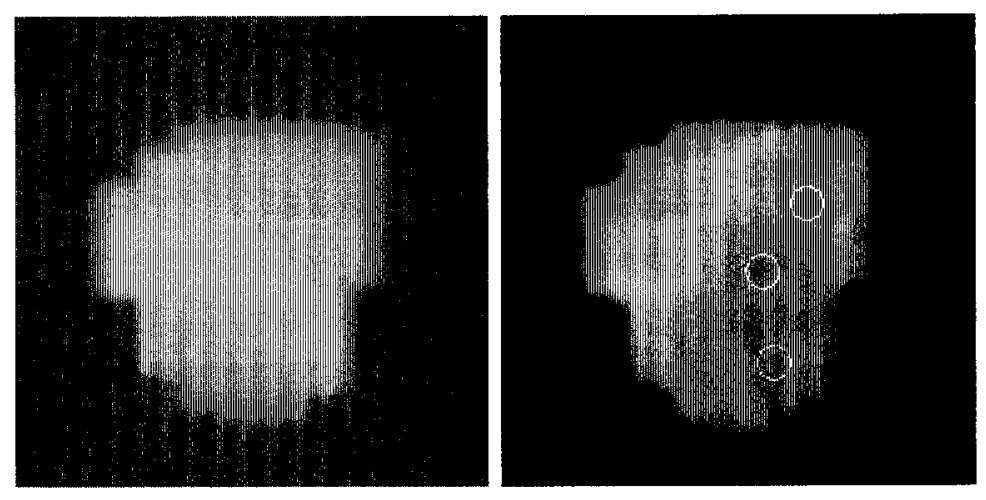

Figure 6.8: Lateral field image with corresponding contrast enhanced image.

Figure 6.9 displays the results using the algorithm presented in this thesis. As predicted, seed 3 next to the bone is not properly detected, however seeds 1 and 2 are discovered accurately. In the first image, the ROI is placed close the field edge causing the standard deviation to maximize near the edge. If the ROI is moved away from the edge, as seen in the second image, the standard deviation now maximizes at the bone location and two disconnected pieces are located both not at the proper seed position. In the third image, the ROI is placed manually so that it does not overlap the bony structures or placed in close proximity to the edge. As anticipated, the third seed is now properly detected. It is safe to say that, if bones are not prevalent in the portal image and the field edges are at a certain distance from the ROI, the seed detection algorithm shows excellent results in the ability to detect fiducial gold seeds 

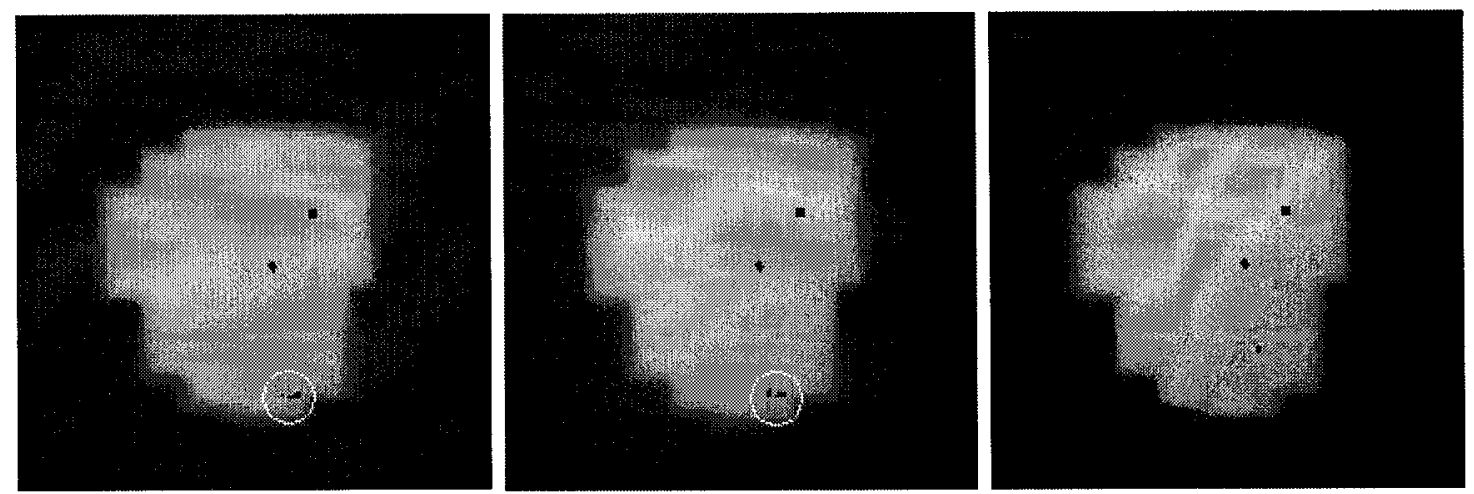

Figure 6.9: Detection of seed 3 placed close to the field edge and the hip bone in a lateral field image.

correctly in electronic portal images.

\subsection{Resources and Measures}

\subsubsection{Space requirements}

The space required in the implementation is very small since the largest data structure contains the blocks information in the ROI; 72 blocks in total. The rest of data structures are with respect to the number of blocks overlapping a seed used in the contour tracing algorithm, the number of pixels included in the seed polygon used in the center of gravity algorithm and, the number of pieces detected as a possible seed (if detected disconnected pieces). Therefore, the data structures are bounded in size by the number of $3 \times 3$ blocks inside the ROI. 


\subsubsection{Running time}

The points below give the complexity of the algorithm steps. Each step is bounded by the number of processed pixels.

1) Standard deviation calculation and finding the maximum, bounded by the number of $3 \times 3$ blocks (at most 72 blocks, each of 9 pixels).

2) Seed intensity range detection, bounded by the number of iterations it takes to converge, for which each iteration looks at 8 surrounding blocks (at most 72 pixels).

3) Geometry verification, bounded by the number of pixels included in the seed polygon (at most 16 pixels) and the number of steps included in the verification process (contour tracing, holes, smallest seed pattern and "line-like" feature detection).

4) CoG calculation, bounded by the number of pixels inside the polygon (at most 16 pixels) and the number of steps included in the CoG calculation (triangulation method bounded by the square of the number of seed pixels, areas and CoGs bounded by the number of triangles).

5) Intra-seed distances calculation, bounded by the total number of seeds - a constant.

Therefore, the algorithms runs in $O(n)+k$ time, for $n$ the number of pixels inside the ROI and $k$ is the number of pixels processed during the CoG calculations $(k<$ $n)$. 


\subsubsection{Clinical Time}

Table 6.4 gives the image number ( 6 images - 18 seeds) with the corresponding running time in seconds the algorithm took to accurately detect all three gold seeds. The algorithm was tested on a PIII, $450 \mathrm{MHz}$ machine. All gold seeds were automatically detected in the portal images well within 1 second.

\begin{tabular}{|c|c|}
\hline Image & Time in s \\
\hline \hline 1 & 0.03 \\
\hline 2 & 0.03 \\
\hline 3 & 0.03 \\
\hline 4 & 0.04 \\
\hline 5 & 0.05 \\
\hline 6 & 0.02 \\
\hline
\end{tabular}

Table 6.4: Running time of the seed detection algorithm in 6 portal images. 


\section{Chapter 7}

\section{Conclusion and Future Work}

\subsection{Conclusion}

A simple, efficient and accurate seed detection algorithm has been developed that automatically detects fiducial seed positions imaged by an EPID. No special image processing techniques are used, which improves the performance of the algorithm. Only simple histogram determination is evoked on a small region of the image, which is in contrast to previous work [30,31, 32, 37]. The steps involve searching for the best suitable seed shape by automatically defining an intensity range for each seed. Error checking consists of three major steps: the seed size, the seed geometry, and the seed patterns (the Euclidean distance between seeds in corresponding image pairs). This method is fast, accurate and efficient making it into a valuable tool in further improving the efficacy and specificity of the radiation treatment of prostate cancer.

\subsection{Future Work}

Future work will involve more testing and preliminary clinical studies before making the algorithm clinically available. The new portal imaging system is currently in the process of being installed at the ORCC. Once the system will be usable, the testing process will become crucial in finalizing the project explained in this thesis. 
The first task will involve testing the accuracy in automatically placing the ROI in portal images using prior knowledge of the location of the seeds identified at treatment planning. For a given set of images, the percentage in which the ROI completely overlaps a seed will be calculated. The results will help in evaluating the correctness of the ROI dimensions and furthermore, verify the claim that a prostate movement over $1 \mathrm{~cm}$ in the cranial-caudal direction is an extreme case.

As mentioned in Chapter 2, the seed size at the ORCC is $0.6 \times 3 \mathrm{~mm}$ and the size used in this thesis is $1.6 \mathrm{~mm} \times 3 \mathrm{~mm}$, a significant difference. If the diameter of the seed stays at $0.6 \mathrm{~mm}$ and the imaging system resolution is $0.55 \mathrm{~mm} / \mathrm{pixel}$ in both the $\mathrm{x}$ and $\mathrm{y}$ directions, the smallest possible seed projection is one pixel. It is highly unlikely that the smallest seed projection will be detectable. The current discussion at the ORCC is to change the seed diameter to $1 \mathrm{~mm}$, permitting the smallest projection of a seed to increase to at least 4 pixels, making the effectiveness of the automatic seed detection algorithm more promising.

To test the case where a seed in the portal image is close to the field edge, the location of the field edge must be known. Therefore, an edge detection method will be applied so that when the ROI is automatically placed, the distance to the edge can be calculated and the ROI moved if necessary.

The patient treatment file containing the leaf positions with respect to the isocenter is accessible at the ORCC. Due to the physical limitations of EPIDs, portal images have poor sharpness. The difficulty lies in accurately defining the edges but with the help of the treatment file the task is simplified [1]. The treatment file contains the height of the field and the distance of each leaf to the isocenter. We therefore have the length of the top and bottom field edges. Once these edges are detected, their lengths and distance between the two edges can be confirmed with the information in the treatment file. The delineation of the MLC can be extracted and compared to the automatic leaf edge detection. Consequently, possible errors in the leaf positions can be detected at treatment time. Figure 7.1 shows the field edge location extracted from the treatment file in red, the yellow dots are the automatic detection of the leaf location. Clearly, the last two MLC leaves on the right side are misplaced resulting in the unpredicted delivery of radiation to nearby tissues. 


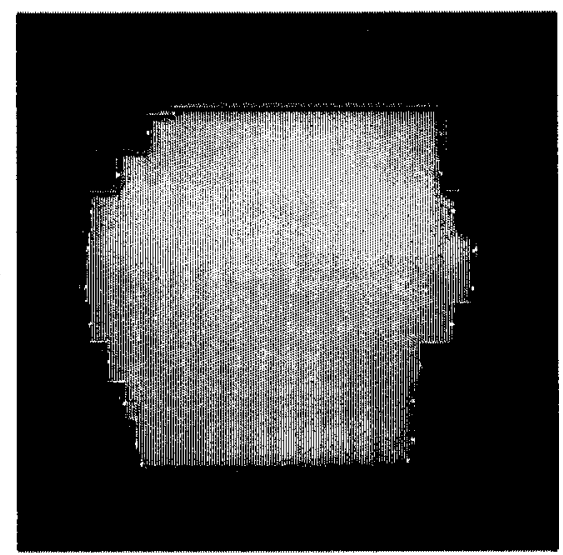

Figure 7.1: Field Edge Detection.

The patient treatment files are on a different file system than those used during treatment time and hence, are not accessible during radiation delivery. Digital Imaging and Communications in Medicine (DICOM) is a standard system used at hospitals for distributing images and associated patient information between different manufactured medical devices [52]. To access the patient's treatment information at radiation delivery time, an automated technique in reading and extracting information from the DICOM files will be integrated into the presented project.

In the near future, the local minima method used in [37] will be compared to the method presented in Chapter 5 (highest standard deviation). It will be interesting to make a comparison between the two methods and testing the differences in seed location. The local minima method may return several possible seed location in each ROI. Intuitively, we expect that our method will be more robust in terms of locating one accurate seed location for each ROI.

In future work, we intend to use of each seed's approximate orientation extracted during treatment planning as seen in $[31,32]$. This a priori information can be extracted with the reconstruction of each field from CT data. At the ORCC, the claim is that the prostate does not rotate significantly between the time of simulation to the time of treatment delivery such that the prior orientation of a seed will be close to the same orientation in the portal image captured at treatment time. If the orientation is known and the distance between points in this direction is calculated, 
we can predict whether or not the projection of a seed is the smallest possible seed projection. The smaller the calculated distance, the closer the projection is to a circle (base of cylinder) and the larger the distance, the closer the projection is to a rectangle (side view of cylinder). This can help in deciding not to smooth the areas which consist of very small seed projection. Furthermore, in order to help with the detection of seeds with smaller diameters than the seeds used in this thesis, some preprocessing algorithms such as CLAHE $[35,36]$ and LoG [15] will be investigated.

As mentioned in Chapter 5 , there are two core requirements that need to be met for this thesis to be clinically acceptable. Requirement 1 has been met since the detection algorithm runs within one second. However, to test requirement 2 in terms of the the accuracy in $\mathrm{mm}$, the prior calculations of the intra-seed distances will be available only once we have access to the new imaging system at the ORCC. 


\section{Bibliography}

[1] V. Audet. Automatic Edge Detection in Medical Images, Honours Project, Carleton University, 1999.

[2] V. Audet, P. Bose, D. Nussbaum, J-R. Sack, J. Szanto and A. Whitehead. Automatic Seed Detection in On-Line Portal Images for Prostate Cancer, Proc. of the Second IASTED International Conference on Visualization, Imaging and Image Processing (VIIP), 2002, 361-366.

[3] J.M. Balter, H.M. Sandler, K. Lam, R.L. Bree, A.S. Lichter and R.K. Ten Haken. Measurement of Prostate Movement over the Course of Routine Radiotherapy using Implanted Markers, Int. J. Radiation Oncology Biol. Phys. 31(1), 1995, 113-118.

[4] J.M. Balter, K.L. Lam, H.M. Sandler, J.F. Littles, R.L. Bree and R.K. Ten Haken. Automated Localization of the Prostate at Time of Treatment using Implanted Radiopaque Markers: Technical Feasibility, Int. J. Radiation Oncology Biol. Phys. 33(5), 1995, 1281-1286.

[5] G.T Barnes. Noise Analysis of Radiographic Imaging, Recent Developments in Digital Imaging, AIP, New York, 1985, 16-38.

[6] C.J. Beard, P. Kijewski, M. Bussière, R. Gelman, D. Gladstone, K. Shaffer, M. Plunkett, P. Costello and N. Coleman. Analysis of Prostate and Seminal Vesicle Motion: Implications for Treatment Planning, Int. J. Radiation Oncology Biol. Phys. 34(2), 1996, 451-458. 
[7] P. Bergström, P-O. Löfroth and A. Widmark. High-Precision Conformal Radiotherapy (HPCRT) of Prostate Cancer - A New Technique for Exact Positioning of the Prostate at the Time of Treatment, Int. J. Radiation Oncology Biol. Phys. 42(2), 1998, 305-311.

[8] A.L. Boyer, L. Antonuk, A. Fenster, M.V. Herk, H. Meertens, P. Munro, L.E. Reinstein and J. Wong. A Review of Electronic Portal Imaging Devices (EPIDs), Medical Physics 19(1), 1992, 1-16.

[9] J. Bijhold, K.G.A. Gilhuijs, M. van Herk and H. Meertens. Radiation Field Edge Detection in Portal Images, Phys. Med. Biol. 36(12), 1991, 1705-1710.

[10] I. Crooks and B.G. Fallone. Contrast Enhancement of Portal Images by Selective Histogram Equalization, Medical Physics 20(1), 1993, 199-204.

[11] J.M. Crook, Y. Raymond, D. Salhani, H. Yang and B. Esche. Prostate Motion during Standard Radiotherapy as Assessed by Fiducial Markers, Radiotherapy and Oncology 37, 1995, 35-42.

[12] K.P. McGee, T.E. Schultheiss and E.E. Martin, A Heuristic Approach to Edge Detection in On-Line Portal Imaging, Int. J. Radiation Oncology Biol. Phys. 32(4), 1995, 1185-1192.

[13] K.G.A. Gilhuijs, A.A.H. El-Gayed, M. van Herk and R.E. Vijlbrief. An Algorithm for Automatic Analysis of Portal Images: Clinical Evaluation for Prostate Treatments, Radiotherapy and Oncology 29, 1993, 261-268.

[14] G. Gluhchev and S. Shalev, Fast Algorithm for Radiation Field Edge Detection, SPIE Vol. 1898 Image Processing, 1993, 296-303.

[15] L.M. Girouard, J. Pouliot, X. Maldague and A. Zaccarin. Automatic Setup Deviation Measurements with Electronic Portal Images for Pelvic Fields, Medical Physics 25(7), 1998, 1180-1185. 
[16] G. Hilt, D. Wolf and P. Aletti. Preprocessing of Control Portal Images for Patient Setup Verification during the Treatments in External Radiotherapy, Medical Physics 26(12), 1999, 2539-2549.

[17] X. Kong, H. Everett and G. Toussaint. The Graham Scan Triangulates Simple Polygons, Pattern Recognition Letters, vol. 11, 1990, 713-716.

[18] K.W. Leszczynski and S. Shalev. Digital Contrast Enhancement for Online Portal Imaging, Med. and Biol. Eng. and Comput., 1989, 507-512.

[19] K.W. Leszczynski, S. Shalev and N.S. Cosby. An Adaptive Technique for Digital Noise Suppression in On-Line Portal Imaging, Phys. Med. Biol. 35, 1990, 429439 .

[20] K.W. Leszczynski, S. Shalev and S. Ryder. A Study on the Efficacy of Digital Enhancement of On-Line Portal Images, Medical Physics 19(4), 1992, 999-1004.

[21] K.W. Leszczynski, S. Shalev and N.S. Cosby. The Enhancement of Radiotherapy Verification Images by an Automated Edge Detection Technique, Medical Physics 19(3), 1992, 611-621.

[22] A. Lubiw. Decomposing Polygonal Regions into Convex Quadrilaterals, Proceedings ACM Symp. on Computational Geometry 1985, 97-106.

[23] S. Malone, J. Szanto, G. Perry, L. Gerig, S. Manion, S. Dahrouge and J. Crook. A Prospective Comparison of Three Systems of Patient Immobilzation for Prostate Radiotherapy, Int. J. Radiation Oncology Biol. Phys. 48(3), 2000, 657-665.

[24] S. Malone, J.M. Crook, W.S. Kendal and J. Szanto. Respiratory-Induced Prostate Motion: Quantification and Characterization. Int. J. Radiation Oncology Biol. Phys. 48(1), 2000, 105-109.

[25] S. Malone, R. Donker, M. Broader, S. Dahrouge, J. Szanto, L. Gerig, G. Bociek and J. Crook. Effects of Urethrography on Prostate Motion: Considerations for Radiotherapy Treatment Planning of Prostate Carcinoma, Int. J. Radiation Oncology Biol. Phys. 46(1), 2000, 89-93. 
[26] S. Malone, R. Donker, S. Dahrouge, L. Eapen, I. Aref, G. Perry and J. Szanto. Treatment Planning Aids in Prostate Cancer: Friend or Foe ?, Int. J. Radiation Oncology Biol. Phys. 51(1), 2001, 49-55.

[27] J. Moseley and P. Munro. Display Equalization: A New Method for Portal Images, Medical Physics 20(1), 1993, 99-102.

[28] P. Munro. Portal Imaging Technology: Past, Present and Future, Semin. Radiat. Oncol. 5(2), 1995, 115-133.

[29] P. Munro. On Line Portal Imaging, 40th Annual Meeting, American Society for Therapeutic Radiology and Oncology, 1998.

[30] A.J. Nederveen, J.J.W. Lagendijk and P. Hofman. Visibility and Detection of Fiducial Gold Markers for On-Line Megavoltage Position Verification, 1999.

[31] A. Nederveen, J. Lagendijk and P. Hofman. Detection of Fiducial Gold Markers for Automatic On-Line Megavoltage Position Verification using a Marker Extraction Kernel (MEK), Int. J. Radiation Oncology Biol. Phys. 47(5), 2000, 1435-1442.

[32] A.J. Nederveen, J. Lagendijk and P. Hofman. Feasibility of Automatic Marker Detection with an a-Si Flat-Panel Imager, Phys. Med. Biol. 46, 2001, 1219-1230.

[33] A.R. Padhani, V.S. Khoo, J. Suckling, J.E. Husband, M.O. Leach and D.P. Dearnaley. Evaluating the Effect of Rectal Distension and Rectal Movement on Prostate Gland Position using Cine MRI, Int. J. Radiation Oncology Biol. Phys. 44(3), 1999, 525-533.

[34] O. Petrascu, A. Bel, N. Linthout, D. Verellen, G. Soete and G. Storme. Automatic On-Line Electronic Portal Image Analysis with a Wavelet-Based Edge Detector, Medical Physics 27(2), 2000, 321-329.

[35] S.M. Pizer, J.D. Austin, J.R. Perry, H.D. Safrit and J.B. Zimmerman. Adaptive Histogram Equalization for Automatic Contrast Enhancement of Medical Images, SPIE Vol. 26 Medicine XIV/PACS IV, 1986, 242-250. 
[36] S.M. Pizer, E.P. Amburn, J.D. Austin, R. Cromartie, A. Geselowitz, T. Greer, B.T.H. Romeny, J.B. Zimmerman and K. Zuiderveld. Adaptive Histogram Equalization and Its Variations, Comp. Vision, Graphics and Image Proc. 39, 1987, 355-368.

[37] S. Pouliot, A. Zaccarin, D. Laurendeau and J. Pouliot. Automatic Detection of Three Radio-Opaque Markers for Prostate Targeting using EPID during External Radiation Therapy, 2001.

[38] R. Rajapakshe and S. Shalev. Noise Analysis in Real-Time Portal Imaging. I. Quantization Noise $^{a)}$, Medical Physics 21(8), 1994, 1263-1268.

[39] R. Rajapakshe and S. Shalev. The Influence of Digitization on Image Noise, Proc. of the Xith Int. Conf. on the use of Computers in Radiation Therapy, 1994, 230-231.

[40] S.A. Rosenthal, M. Roach III, B.J. Goldsmith, E.C. Doggett, B. Pickett. H-S. Yuo, E.M. Soffen, R.L. Stern and J.K. Ryu. Immobilization Improves the Reproducibility of Patient Positioning during Six-Field Conformal Radiation Therapy for Prostate Carcinoma, Int. J. Radiation Oncology Biol. Phys. 27, 1993, 921926.

[41] J.-C. Rosenwald, O. Dahl, G. Gaboriaud, D. Pontvert, A. Brahme, F. Nüsslin, W. De Neve, C. Kappas, B. Lind and C. de Wagter. Classification of Conformal Radiotherapy, a proposal from the Dynarad European Group submitted to Radiothearpy and Oncology, 1998.

(www.isis3d.com/i3d/pages/articles/class.html)

[42] J.-R. Sack and G.T. Toussaint. Guard Placement in Rectilinear Polygons, Computational Morphology, Mach. Int. and Pattern Recog. 6, 1988, 153-175.

[43] H.M. Sandler, R.L. Bree, P.W. McLaughlin, H.B. Grossman and A.S. Lichter. Localization of the Prostatic Apex for Radiation Therapy using Implanted Markers. Int. J. Radiation Oncology Biol. Phys. 27, 1993, 915-919. 
[44] S. Shalev. On-Line Portal Imaging: Contributions and Limitations in Clinical Practice, "3-D Conformal Radiotherapy", Frontiers in Radiation Therapy and Oncology, 1996, 156-167.

[45] G. Toussaint. Chapter 2: Grids, Connectivity and Contour Tracing. (www-cgrl.cs.mcgill.ca/ godfried/teaching/pr-notes/contour.ps)

[46] E. Vigneault, J. Pouliot, J. Lavardière, J. Roy and M. Dorion. Electronic Portal Imaging Device Detection of Radioopaque Markers for the Evaluation of Prostate Position during Magavoltage Irradiation: A Clinical Study. Int. J. Radiation Oncology Biol. Phys. 37(1), 1997, 205-212.

[47] F.M. Vos, J. Stoeckel, P.H. Vos and A.M. Vossepoel. Evaluation of an Automatic System for Simulator/Portal Image Matching, MICCAI, 2000, 442-451.

[48] H. Wang and B.G. Fallone. A Robust Morphological Algorithm for Automatic Radiation Field Extraction and Correlation of Portal Images, Medical Physics 21(2), 1994, 237-244.

[49] B. Wowk and S. Shalev. Thick Phosphor Screens for On-Line Portal Imaging, Medical Physics 21(8), 1994, 1269-1276

[50] www.prostate.com

[51] www.saltspring.com/brochman/math/centroid/centroid.html

[52] medical.nema.org/dicom/2001.html 